International Journal of Applied Electromagnetics and Mechanics (Japan)

Impact factor $=0.684$

Editor-in-Chief: Prof. Dr. Toshiyuki Takagi, Institute of Fluid Science, Tohoku University

Katahira 2-1-1, Aoba-ku, Sendai 980-8577, Japan.

https://content.iospress.com/iournals/international-journal-of-applied-electromagnetics-and-mechanics/63/2

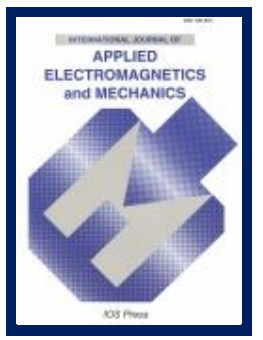

(Accepted July $27^{\text {th }}$ 2020)

\title{
UNSTEADY NONLINEAR MAGNETOHYDRODYNAMIC MICROPOLAR TRANSPORT PHENOMENA WITH HALL AND ION-SLIP CURRENT EFFECTS: NUMERICAL STUDY
}

O. Anwar Bég ${ }^{1}$, Tasveer A. Bég ${ }^{2}$, M. Ferdows ${ }^{3}$, B. Vasu ${ }^{4}$, Ali Kadir ${ }^{1}$, H. J. Leonard ${ }^{1}$ and S. Kuharat ${ }^{1}$ ${ }^{1}$ Multi-Physical Engineering Sciences Group, Mechanical Engineering Department, School of Science, Engineering and Environment (SEE), University of Salford, Manchester, UK.

${ }^{2}$ Engineering Mechanics Research, Israfil House, Dickenson Rd., Manchester, M13, UK. ${ }^{3}$ Fluid Dynamics, College of Engineering, Louisiana State University, Ruston, Louisiana, USA.

${ }^{4}$ Department of Mathematics, MNNIT Allahabad, Prayagraj, Uttar Pradesh-211004, India.

* Corresponding Author - Email: bvasu@mnnit.ac.in

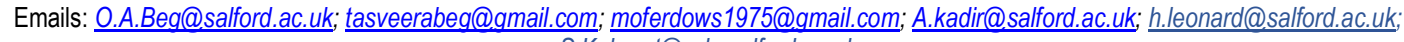
S.Kuharat@edu.salford.ac.uk

\begin{abstract}
:
Unsteady viscous two-dimensional magnetohydrodynamic micropolar flow, heat and mass transfer from an infinite vertical surface with Hall and lon-slip currents is investigated theoretically and numerically. The simulation presented is motivated by electro-conductive polymer (ECP) materials processing in which multiple electromagnetic effects arise. The primitive boundary layer conservation equations are transform ed into a non-similar system of coupled non-dimensional momentum, angular momentum, energy and concentration equations, with appropriate boundary conditions. The resulting two-point boundary value problem is solved numerically by an exceptionally stable and welltested implicit finite difference technique. A stability analysis is included for restrictions of the implicit finite difference method (FDM) employed. Validation with a Galerkin finite element method (FEM) technique is included. The influence of various parameters is presented graphically on primary and secondary shear stress, Nusselt number, Sherwood number and wall couple stress. Secondary (cross flow) shear stress is strongly enhanced with greater magnetic parameter (Hartmann number) and micropolar wall couple stress is also weakly enhanced for small time values with Hartmann number. Increasing thermo-diffusive Soret number suppresses both Nusselt and Sherwood numbers whereas it elevates both primary and secondary shear stress and at larger time values also increases the couple stress. Secondary shear stress is strongly boosted with Hall parameter. Ion slip effect induces a weak modification in primary and secondary shear stress distributions. The present study is relevant to electroconductive non-Newtonian (magnetic polymer) materials processing systems.
\end{abstract}

KEYWORDS: Micropolar fluid; Hall and lon-slip current; Thermal diffusion; Species diffusion; wall couple stress; primary and secondary shear stress; magnetic field; implicit finite difference method (FDM); electroconductive polymer processing; Galerkin finite element method (FEM).

\section{INTRODUCTION}

Electromagnetic flows of both Newtonian and non-Newtonian fluids continue to attract the attention of researchers owing to ever-growing applications in aerodynamics and propulsion systems [1,2], materials processing [3-5], rotating disk reactor and trickle-bed chemical engineering technologies $[6,7]$ and novel biomedical engineering developments [8,9]. Electromagnetic flows are frequently accompanied with heat and mass transfer and are of interest due to the proven ability of magnetic fields in controlling and manipulating performance of electrically conducting liquids [10]. Numerous theoretical and computational studies of such flows have been communicated in the past two decades 
using a variety of magnetic non-Newtonian models including Walters-B models [11], couple stress models [12], Oldroyd-B models [13] and Casson models [14]. Among the diverse range of rheological models available, the micropolar model introduced by Eringen [15] has certain exceptional features which allow the simulation of micro-structural effects in non-Newtonian fluids. Micropolar fluids are able to support local stress moment, body moments and are influenced by the microelement spin inertia. Micropolar fluids, a sub-class of the more general micromorphic fluid [16], can therefore represent real engineering fluids consisting of rigid, randomly oriented or spherical particles suspended in a viscous medium in which the deformation of the particles is ignored. This model is very useful for simulating certain electro-conductive polymers, food stuffs, detergents, gel propellants, colloidal suspensions, liquid crystals, lubricants, bubbly liquids, paints and physiological fluids. The theory of micropolar fluids has been a field of active research in fluid mechanics, for nearly five decades. Magnetohydrodynamic studies of micropolar flows have received considerable attention in recent years and researchers have utilized a diverse range of numerical algorithms to solve these nonlinear boundary value problems. Bég et al. [17] employed the network simulation electro-thermal code, PSPICE, to analyze magnetohydrodynamic thin film flows of micropolar fluids along oblique surfaces. Eldabe et al. [18] used a Chebyshev spectral finite difference method to analyze hydromagnetic micropolar convection boundary layer flow from an extending polymeric sheet. Bég et al. [19] used a homotopy algorithm with symbolic software to simulate unsteady magneto-micropolar squeeze film hydrodynamics. Rawat et al. [20] applied a variational finite element method to simulate unsteady hydromagnetic free convection heat and mass transfer in porous media with variable thermophysical properties. Further studies have been communicated by Hayat et al. [21], Kumar et al. [22] and Haque and Alam [23], the latter also examining Ohmic and viscous dissipation effects.

In the above studies generally, weak magnetic fields have been considered, for which Hall and ionslip currents may be neglected. However, in certain manufacturing processes [24], very strong magnetic fields are deployed and as such both Hall current and ionslip effects become important. With the application of a strong magnetic field, a significant voltage difference (or Hall voltage) is generated across an electric conductor (working fluid) where the magnetic field is perpendicular to the current. The Hall parameter is significant when the magnetic field is very high or when the collision frequency is very low. Certain materials e.g. electro-conductive polymers become highly conducting under ionization. Therefore, Hall currents may exert a critical role in the behaviour of such fluid materials. Several researchers have studied magnetohydrodynamic convection heat transfer (and mass transfer) flows with Hall current and/or ionslip effects. Interesting analyses include Datta and Mazumder [25], Ram [26], Raptis and Kafoussias [27] and Sattar and Hossain [28]. More recent studies of hydromagnetic flows with Hall and ionslip effects include Bég et al. [29] who studied hydromagnetic 
rotating channel flow in inclined magnetic fields and porous media using network simulation, Zueco et al. [30] who also considered Joule heating, Ghosh et al. [31] who considered Ekman number effects and Zueco et al. [32] who examined dusty fluids. These studies did not consider micropolar fluids which have received very little attention in Hall and ionslip magnetohydrodynamics. In the present investigation, therefore we consider transient ionized micropolar convection heat and mass transfer flow with the influence of Hall and Ion-slip currents. Viscous dissipation and Joule heating (Ohmic dissipation) effects are also considered as are thermo-diffusion (Soret) effect. The presence of Hall currents generates a secondary (cross) flow and therefore a two-dimensional formulation is necessary. The governing unsteady two-dimensional partial differential conservation equations are transformed into a dimensionless system of non-similar non-linear partial differential equations which are solved by implicit finite difference method (FDM). A numerical stability analysis is also conducted. The influence of micropolar rheological and other thermophysical parameters on dimensionless primary and secondary shear stresses, couple stress (dimensionless wall micro-rotation gradient), Nusselt number (dimensionless wall heat transfer rate) and Sherwood number (dimensionless wall mass transfer rate) are examined in detail. Validation of solutions is also included using a Galerkin finite element method (FEM). Although many commercial codes are available for multi-physical electromagnetic modelling e.g. COMSOL Multiphysics, ANSYS Maxwell, ADINA, STAR-CCM etc, which are excellent for simulating coupled fluid and electromagnetics, nevertheless they are confined to very simple material fluid models e.g. Newtonian, Carreau fluid, power-law fluid. They do not feature a non-Newtonian routine or solver for micropolar fluids. i.e. they do not provide a solver for micromorphic (angular micro-spin) and this is why researchers worldwide are still compelled to use their own programs to solve micropolar hydromagnetic flows. As such in the present study therefore a finite difference method code which has been extensively validated is adopted in addition to a finite element code for verification. The present study is relevant to electromagnetic thermal processing of conducting polymers.

\section{MATHEMATICAL ELECTROMAGNETIC MICROPOLAR FLOW MODEL}

We consider unsteady free convection heat and mass transfer from an ionized micropolar fluid along an infinite vertical surface (located at $y=0$ ). The vertical surface is an electrically non-conducting plate oriented along the $x$-axis in the upward direction. A uniform magnetic field of strength, $B_{o}$, acts along the positive $y$-direction, normal to the plate. Hall and ion slip effects are present, and the applied magnetic field is strong [33, 34]. From the Gauss equation of conservation of charge $\nabla \bullet J=0$ (where $J$ denotes current density) and this yields $J_{y}=0$ i.e. electric current vanishes at the plate and everywhere else. The magnetic Reynolds number is sufficiently small to neglect magnetic induction 
effects. The physical configuration for the flow regime is depicted in Fig. 1. The late is permeable and a uniform suction velocity, $v_{0}$ acts in the $y$-direction. The plate is assumed to be of infinite extent and the fluid motion is unsteady so that all the flow variables are dependent only on the $y$-coordinate and time, $t$. The presence of Hall currents induces a cross flow in the $z$-direction. Therefore, flow occurs in the $y-z$ plane and requires two momentum conservation equations for its accurate description. In accordance with the above assumptions and invoking the Boussinesq approximation, following Ghosh et al. [35], the boundary layer equations for linear momentum, angular momentum, heat and species diffusion, for the flow may be defined as follows:

\section{Primary linear momentum conservation (y-direction):}

$$
\frac{\partial u}{\partial t}-v_{o} \frac{\partial u}{\partial y}=g \beta\left(T-T_{\infty}\right)+g \beta^{*}\left(C-C_{\infty}\right)+\left(\vartheta+\frac{K}{\rho}\right) \frac{\partial^{2} u}{\partial y^{2}}+\frac{K}{\rho} \frac{\partial N}{\partial y}-\frac{1}{\rho} \frac{B_{o}{ }^{2} \sigma_{e}}{\left(\alpha_{e}{ }^{2}+\beta_{e}{ }^{2}\right)}\left(\alpha_{e} u+\beta_{e} w\right)
$$

Secondary linear momentum conservation (z-direction):

$$
\frac{\partial w}{\partial t}-v_{o} \frac{\partial w}{\partial y}=\left(\vartheta+\frac{K}{\rho}\right) \frac{\partial^{2} w}{\partial y^{2}}+\frac{1}{\rho} \frac{B_{o}{ }^{2} \sigma_{e}}{\left(\alpha_{e}{ }^{2}+\beta_{e}{ }^{2}\right)}\left(\beta_{e} u-\alpha_{e} w\right)
$$

Angular momentum conservation:

$$
\frac{\partial N}{\partial t}-v_{o} \frac{\partial N}{\partial y}=\frac{\gamma}{\rho j} \frac{\partial^{2} N}{\partial y^{2}}-\frac{K}{\rho j} \frac{\partial u}{\partial y}-2 \frac{K}{\rho j} N
$$

\section{Energy (heat) conservation:}

$\frac{\partial T}{\partial t}-v_{0} \frac{\partial T}{\partial y}=\frac{k}{\rho C_{p}}\left(\frac{\partial^{2} T}{\partial y^{2}}\right)+\frac{1}{C_{P}}\left(\vartheta+\frac{K}{\rho}\right)\left[\left(\frac{\partial u}{\partial y}\right)^{2}+\left(\frac{\partial w}{\partial y}\right)^{2}\right]+\frac{\sigma_{e} B_{0}^{2}}{\rho C_{\rho}}\left(u^{2}+w^{2}\right)$

\section{Species diffusion (concentration) conservation}

$$
\frac{\partial C}{\partial t}-v_{0} \frac{\partial C}{\partial y}=D_{m}\left(\frac{\partial^{2} C}{\partial y^{2}}\right)+D_{T} \frac{K_{T}}{T_{m}} \frac{\partial^{2} T}{\partial y^{2}}
$$

The boundary conditions for the present problem are given by:

$$
\begin{aligned}
& u=0, w=0, N=-s \frac{\partial u}{\partial y}, T=T_{\omega}, C=C_{w} \text { at } y=0 \\
& u=0, w=0, N=0, T \rightarrow T_{\infty}, C \rightarrow C_{\infty} \text { at } y=\infty
\end{aligned}
$$

Here $u, v, w$ are the velocity components in the $x, y, z$ direction respectively, $g$ is the acceleration due to gravity, $\beta$ is the coefficient of volume expansion, $\beta^{*}$ is the volumetric coefficient of expansion with concentration, $T$ and $T_{\infty}$ are the temperature of the fluid inside the thermal boundary layer and the fluid temperature in the free stream, respectively while $C$ and $C_{\infty}$ are the corresponding concentrations. $\vartheta$ is the kinematic Newtonian viscosity, $K$ is the Eringen vortex viscosity, $\rho$ is micropolar fluid density, $N$ is the micro-rotation (angular velocity), $\sigma_{e}$ is the electrical conductivity, $B_{o}$ 
is the strength of the applied magnetic field, $\beta_{e}$ is the Hall parameter, $\alpha_{e}=1+\beta_{i} \beta_{e}$ (where $\beta_{i}$ is the ionslip parameter), $\gamma$ is the spin gradient viscosity, $j$ is the micro-inertia per unit mass, $\kappa$ is the thermal conductivity of the medium, $c_{p}$ is the specific heat at constant pressure, $c_{s}$ is concentration susceptibility, $D_{m}$ is the coefficient of mass diffusivity, $D_{T}$ is the coefficient of thermal diffusivity, $K_{T}$ is thermal diffusion rate, $T_{m}$ denotes mean fluid temperature, $t$ is dimensional time, $s$ is an arbitrary micropolar wall constant. The last parameter here warrants some elaboration. The $s$ parameter controls the wall micro-rotation boundary conditions in eqn. (6). With $s=0$, then $N=0$ and this corresponds to the no-spin condition i.e., the microelements in a concentrated particle flow close to the wall are not able to rotate. The case $s=0.5$ is associated with the vanishing of the anti-symmetric part of the stress tensor and represents weak concentrations of micropolar fluid micro-elements. In a fine particulate suspension, the particle spin is equal to the fluid velocity at the wall. Finally, the case $s=1$, denotes the transitional or turbulent boundary layer flow case, which is not relevant to the present laminar flow simulations. In the wall boundary condition (6), the constant $v_{0}$ represents a suction/injection velocity (positive for suction, negative for injection). The primitive form of the transport model defined by Eqns. (1)-(6) is challenging to solve, even with numerical methods. It is advantageous to normalize the model which eliminates the second spatial variable $(z)$ and simultaneously introduces dimensionless thermo-physical parameters which allow the fluid dynamics to be more easily studied. Proceeding with the analysis we introduce the following non-dimensional variables to facilitate a subsequent numerical solution of the two-point non-linear partial differential equation boundary value problem.

$\mathrm{Y}=\frac{\mathrm{yU}_{0}}{\vartheta}, \mathrm{U}=\frac{\mathrm{u}}{\mathrm{U}_{0}}, \mathrm{~W}=\frac{\mathrm{w}}{\mathrm{U}_{0}}, \tau=\frac{\mathrm{tU}_{0}{ }^{2}}{\vartheta}, \Omega=\frac{\mathrm{N} \vartheta}{\mathrm{U}_{0}{ }^{2}}, \theta=\frac{\mathrm{T}-\mathrm{T}_{\infty}}{\mathrm{T}_{\mathrm{W}}-\mathrm{T}_{\infty}}, \Phi=\frac{\mathrm{C}-\mathrm{C}_{\infty}}{\mathrm{C}_{\mathrm{W}}-\mathrm{C}_{\infty}}$

where $Y$ is dimensionless y-coordinate, $U$ is dimensionless $x$-direction velocity (primary velocity), $W$ is dimensionless $z$-direction (cross flow i.e. secondary) velocity, $\tau$ is non-dimensional time, $\Omega$ is dimensionless angular velocity, $\theta$ is dimensionless temperature, $\Phi$ is dimensionless species concentration and $U_{0}$ is characteristic velocity. Introducing the non-dimensional variables (7) in equations (1) to (5), the transformed conservation equations describing the regime reduce to the following form:

$$
\begin{aligned}
& \frac{\partial U}{\partial \tau}-\epsilon \frac{\partial U}{\partial Y}=(1+\Delta) \frac{\partial^{2} U}{\partial Y^{2}}+\Delta \frac{\partial \Omega}{\partial Y}+G_{r} \theta+G_{r} \Phi-\frac{M}{\left(\alpha_{e}^{2}+\beta_{e}^{2}\right)}\left(\alpha_{e} U+\beta_{e} W\right) \\
& \frac{\partial W}{\partial \tau}-\epsilon \frac{\partial W}{\partial Y}=(1+\Delta) \frac{\partial^{2} W}{\partial Y^{2}}+\left(\frac{M}{\alpha_{e}^{2}+\beta_{e}^{2}}\right)\left(\beta_{e} U-\alpha_{e} W\right) \\
& \frac{\partial \Omega}{\partial \tau}-\varepsilon \frac{\partial \Omega}{\partial Y}=\Lambda \frac{\partial^{2} \Omega}{\partial Y^{2}}-\lambda\left(2 \Omega+\frac{\partial U}{\partial Y}\right) \\
& \frac{\partial \theta}{\partial \tau}-\varepsilon \frac{\partial \theta}{\partial Y}=\frac{1}{P_{r}} \frac{\partial^{2} \theta}{\partial Y^{2}}+(1+\Delta) E_{C}\left[\left(\frac{\partial U}{\partial Y}\right)^{2}+\left(\frac{\partial W}{\partial y}\right)^{2}\right]+M \frac{E_{c}}{\left(\alpha_{e}^{2}+\beta_{e}^{2}\right)}\left(U^{2}+W^{2}\right)
\end{aligned}
$$


$\frac{\partial \Phi}{\partial \tau}-\epsilon \frac{\partial \Phi}{\partial Y}=\frac{1}{s_{c}} \frac{\partial^{2} \Phi}{\partial Y^{2}}+s_{0} \frac{\partial^{2} \theta}{\partial Y^{2}}$

The corresponding transformed initial and boundary conditions are:

$U=0, W=0, \Omega=-S \frac{\partial U}{\partial Y}, \theta=1, \Phi=1 \quad$ at $Y=0$

$U=0, W=0, \Omega=0, \theta=0, \Phi=1 \quad$ as $Y \rightarrow \infty$

Here $\varepsilon$ is the transpiration parameter $\left(\varepsilon=\frac{v_{o}}{U_{o}}\right.$ and this parameter $>0$ for suction, $<0$ for injection, and equal to zero for a solid plate), $\Delta$ is the Eringen micropolar material parameter or vortex viscosity parameter and is defined as $\frac{\kappa}{\rho \vartheta}$ ), $G r$ is thermal Grashof number, $M$ is the magnetohydrodynamic parameter, $G r^{*}$ is species Grashof number, $\Lambda$ is the spin gradient viscosity parameter $=\frac{\gamma}{\rho j \vartheta}, \lambda$ is the dimensionless vortex viscosity parameter. Also, $P_{r}$ is Prandtl number, $S_{c}$ is Schmidt number, $S_{o}$ denotes Soret number and $E_{c}$ represents the Eckert number, and these parameters assume their usual definitions - see references $[20,33]$.

\section{NUMERICAL SOLUTION WITH FINITE DIFFERENCE METHOD (FDM)}

We seek a robust numerical solution to the boundary value problem defined by eqns. (8)-(12) under conditions (13). Each partial differential equation is a second order coupled dimensionless equation in two independent variables $(Y, \tau)$. For simplicity the implicit finite difference method has been used. This method has also been employed recently by the authors to solve a number of complex multiphysical problems and the reader is referred to [36]-[38]. It is very efficient and has been benchmarked with many published results. Confidence in the FDM code is therefore high. However, to provide further confirmation of the accuracy in the next section an FEM validation is presented for the entire model. In FDM the region within the boundary layer is discretized as depicted in Fig. $\mathbf{2}$.

It is assumed that the maximum length of boundary layer is $Y_{\max }=30$ which corresponds to $Y$ at infinity. After experimentation with the finite difference mesh, a grid spacing in the $Y$-direction is selected as $m=200$. Hence the constant mesh size along the $Y$-axis is $\Delta Y=0.15(0 \leq Y \leq 30)$. The time variable is discretized with a time step of $\Delta \tau=0.01$, ensuring excellent convergence of solutions. Let $U_{i}^{n}, W_{i}^{n}, \Omega_{i}^{n}, \theta_{i}^{n}$ and $\Phi_{i}^{n}$ denote the values of $U, W, \Omega, \theta, \Phi$ at the end of a time-step respectively. Using the implicit finite difference approximations, the eqns. (8)-(12) assume the algorithmic form: 


\section{Primary Momentum}

$\frac{U_{i}^{n+1}-U_{i}^{n}}{\Delta \tau}-\epsilon \frac{U_{i+1}^{n}-U_{i}^{n}}{\Delta Y}=(1+\Delta) \frac{U_{i+1}^{n}-2 U_{i}^{n}+U_{i-1}^{n}}{(\Delta Y)^{2}}$

$\Delta \frac{\Omega_{i+1}^{n}-\Omega_{i}^{n}}{\Delta Y}+G_{r} \theta_{i}^{n}+G_{r}^{*} \Phi_{i}^{n}-\frac{M}{\left(\alpha_{e}^{2}-\beta_{e}^{2}\right)}\left(\alpha_{e} U_{i}^{n}+\beta_{e} W_{i}^{n}\right)$

\section{Secondary Momentum}

$\frac{W_{i}^{n+1}-W_{i}^{n}}{\Delta \tau}-\epsilon \frac{W_{i+1}^{n}-W_{i}^{n}}{\Delta Y}=(1+\Delta) \frac{W_{i+1}^{n}-2 W_{i}^{n}+W_{i-1}^{n}}{(\Delta Y)^{2}}+\frac{M}{\left(\alpha_{e}^{2}+\beta_{e}^{2}\right)}\left(\beta_{e} U_{i}^{n}-2 \alpha_{e} W_{i}^{n}\right)$

Angular Momentum (Micro-rotation)

$\frac{\Omega_{i}^{n+1}-\Omega_{i}^{n}}{\Delta \tau}-\varepsilon \frac{\Omega_{i+1}^{n}-\Omega_{i}^{n}}{\Delta Y}=\Lambda \frac{\Omega_{i+1}^{n}-2 \Omega_{i}^{n}+\Omega_{i-1}^{n}}{(\Delta Y)^{2}}-\lambda\left[2 \Omega_{i}^{n} \frac{U_{i+1}^{n}-U_{i}^{n}}{\Delta Y}\right]$

Energy

$\frac{\theta_{i}^{n+1}-\theta_{i}^{n}}{\Delta \tau}-\epsilon \frac{\theta_{i+1}^{n}-\theta_{i}^{n}}{\Delta Y}=\frac{1}{p_{\Gamma}} \frac{\theta_{i+1}^{n}-2 \theta_{i}^{n}+\theta_{i-1}^{n}}{(\Delta Y)^{2}}+(1+\Delta) E_{c}\left[\left(\frac{\cup_{i}^{n}+1-U_{i}^{n}}{\Delta Y}\right)^{2}+\left(\frac{W_{i+1}^{n}-W_{i}^{n}}{\Delta Y}\right)^{2}\right]+$

$M \frac{E c}{\left(\alpha_{e}^{2}+\beta_{e}^{2}\right)}\left(U_{i}^{n^{2}}+W_{i}^{n^{2}}\right)$

\section{Species (Concentration)}

$\frac{\Phi_{i}^{n+1}-\Phi_{i}^{n}}{\Delta \tau}-\epsilon \frac{\Phi_{i+1}^{n}-\Phi_{i}^{n}}{\Delta Y}=\frac{1}{S_{C}} \frac{\Phi_{i+1}^{n}-2 \Phi_{i}^{n}+\Phi_{i-1}^{n}}{(\Delta Y)^{2}}+S_{o} \frac{\theta_{i+1}^{n}-2 \theta_{i}^{n}+\theta_{i-1}^{n}}{(\Delta Y)^{2}}$

At the end of any time step $\Delta \tau$, the new temperature $\theta_{i}^{n+1}$, the new concentration $\Phi_{i}^{n+1}$, the new secondary velocity $W_{i}^{n+1}$, the new angular velocity $\Omega_{i}^{n+1}$ and the new primary velocity $U_{i}^{n+1}$ at all interior nodal points, are obtained by successive applications of the above difference equations, respectively. This process is repeated in time and $U_{i}^{n}, W_{i}^{n}, \Omega_{i}^{n}, \theta_{i}^{n}$ and $\Phi_{i}^{n}$, eventually all converge to values which approximate the solution of the master dimensionless equation. Also, the numerical values of the shear stresses, couple stress, Nusselt number and Sherwood number are evaluated by five-point approximate formula for the derivatives. The stability conditions of the implicit finite difference method are given by:

Primary and secondary momentum:

$\epsilon \frac{\Delta \tau}{\Delta Y}+\Lambda \frac{2 \Delta \tau}{(\Delta Y)^{2}}+\lambda \Delta \tau \leq 1$

\section{Energy:}


$\epsilon \frac{\Delta \tau}{\Delta Y}+\frac{2}{P_{r}} \frac{\Delta \tau}{(\Delta Y)^{2}} \leq 1$

\section{Species (Concentration)}

$\frac{2}{S_{c}} \frac{\Delta \tau}{(\Delta Y)^{2}}-\epsilon \frac{\Delta \tau}{\Delta Y} \leq 1$

Here when $\Delta \tau$ and $\Delta Y$ approach zero, the problem attains convergence. This implies that the results of the implicit finite difference method approach the actual solutions.

\section{VALIDATION WITH VARIATIONAL FINITE ELEMENT METHOD}

Since the present model constitutes a significant extension to the existing literature, it is not possible to validate it comprehensively with earlier studies without neglecting many important effects. Therefore, verification of the solutions is conducted via a variational finite element method code based on a weighted residual approach and the Galerkin finite element method. This methodology has been deployed extensively by the authors in recent years in time-dependent micropolar transport phenomena in magnetic materials processing. Relevant examples include Gupta et al. [39] (on mixed convection contracting sheet micropolar flow), Shamshuddin et al. [40] (transient chemically reacting hydromagnetic dissipative micropolar thermosolutal flow from a tilted surface), Shamshuddin et al. [41] (unsteady magnetohydrodynamic heat and mass transfer from a rotating surface in porous media) and Shamshuddin et al. [42] (periodic thermosolutal reactive micropolar flow). The fundamental steps involved in the finite-element analysis are summarized in [39]-[42] and not repeated here. FDM uses numerical differentiation whereas FEM uses numerical integration. Generally, the latter is more accurate for nonlinear problems, although it has been used here also for validation of the FDM solutions. The finite-difference method is the most direct approach to discretizing partial differential equations. A point in space is considered where one takes the continuum representation of the equations and replaces it with a set of discrete equations, called finite-difference equations. The finite-difference method is typically defined on a regular grid and this fact can be used for very efficient solution methods. The method is therefore not usually used for irregular engineering geometries, but more often for rectangular or block-shaped models. Certain formulations of the finite-element method defined on a regular grid are identical to a finite-difference method on the same grid. Regular grids can often be used in meteorological, seismological, and astrophysical simulations, for example. FEM is generally much more popular for complex geometries. However, FDM still remains a powerful and very adaptive tool for electromagnetic fluid dynamics problems. The authors have extensively used both approaches and indeed many others including network electrothermal simulation (PSPICE), Adomian decomposition, smoothed particle hydrodynamics (SPH), Lattice Boltzmann methods (LBM) for magnetofluid dynamics problems in 
recent years. However, for micropolar magnetohydrodynamics, the most versatile approach is undeniably the finite difference method which is therefore adopted in the present study. Proceeding with the finite element formulation, two-node linear elements are deployed. Arbitrary test (interpolation) functions $\Pi_{1}, \Pi_{2}, \Pi_{3}, \Pi_{4}$ and $\Pi_{5}$, are prescribed for the variations in $U, W, \Omega, \theta, \Phi$ respectively. After dropping the order of integration and non-linearity, variational forms are derived for each of the conservation equations (8)-(12). The finite element model may be obtained from these new equations by substituting finite element approximations of the form:

$U=\sum_{j=1}^{2} U_{j}^{e} \psi_{j}^{e}, V=\sum_{j=1}^{2} V_{j}^{e} \psi_{j}^{e}, \Omega=\sum_{j=1}^{2} \Omega_{j}^{e} \psi_{j}^{e}, \theta=\sum_{j=1}^{2} \theta_{j}^{e} \psi_{j}^{e}, \Phi=\sum_{j=1}^{2} \Phi_{j}^{e} \psi_{j}^{e}$

Here $\Pi_{1}=\Pi_{2}=\Pi_{3}=\Pi_{4}=\Pi_{5}=\psi_{j}^{e}(i=1,2)$, where $U_{j}^{e}, W_{j}^{e}, \Omega_{j}^{e}, \theta_{j}^{e}$ and $\Phi_{j}^{e}$ are the primary velocity, secondary velocity, micro-rotation (angular velocity), temperature and species concentration values, respectively, at the $j^{\text {th }}$ node of typical $e^{\text {th }}$ element $\left(Y_{e}, Y_{e+1}\right)$ and $\psi_{i}^{e}$ are the finite element shape functions for this element $\left(Y_{e}, Y_{e+1}\right)$ which are taken as:

$\psi_{1}^{e}=\frac{Y_{e+1}-Y}{Y_{e+1}-Y_{e}}$ and $\psi_{2}^{e}=\frac{Y-Y_{e}}{Y_{e+1}-Y_{e}}, Y_{e} \leq Y \leq Y_{e+1}$

The finite element model of the equations for the $e^{\text {th }}$ element is a $5^{\text {th }}$ order matrix system in which $\left\{\left[K^{m n}\right],\left[M^{m n}\right]\right\}$ and $\left\{\left\{U^{e}\right\},\left\{W^{e}\right\},\left\{\Omega^{e}\right\}\left\{\theta^{e}\right\},\left\{\Phi^{e}\right\},\left\{U^{\prime e}\right\},\left\{W^{\prime e}\right\},\left\{\Omega^{\prime e}\right\},\left\{\theta^{\prime e}\right\},\left\{\Phi^{\prime e}\right\}\right.$ and $\left.\left\{b^{m e}\right\}\right\}(m, n=$ $1,2,3,4,5)$ are the set of matrices of order $2 \times 2$ and $2 \times 1$ respectively and prime () indicates $\frac{d}{d Y}$. The matrices definitions are omitted for brevity. Here the entire flow domain is discretized into successively sized grids of order 121x121 after grid independence tests on more coarse (i.e. less refined) meshes ( $61 \times 61,81 \times 81,101 \times 101)$ in the $Y$ - direction. All the computations are executed with 121 intervals of equal step size 0.01 . At each node, 5 functions are to be evaluated and after assembly of the element equations, a set of 605 non-linear equations are obtained which necessitate an iterative solution subject to the specified boundary conditions. The iterative process is terminated when the following condition is met: $\sum_{i, j}\left|\chi^{n+1}-\chi^{n}\right| \leq 10^{-6}$ where $\chi=U, V, \Omega, \theta, \Phi$ and $n$ denotes the iterative step. Comparison with the FDM solutions is visualized in Figs. 3-4 for primary shear stress $\left(\tau_{x}\right)$, secondary shear stress $\left(\tau_{z}\right)$ with $M=0.03$ (weak magnetic field). Excellent correlation is achieved testifying to the accuracy of the FDM code.

Inspection of Figs. 3 to 4 which depict the effect of magnetic parameter (Hartmann number, $M$ ) on the steady-state shear stresses indicate that the primary flow (Fig. 3) as simulated via primary shear stress at the plate) is decreased with the magnetic parameter, $M$, since magnetic field imposed 
generates a Lorentz damping force which has a decelerating effect. Therefore, minimal shear stress corresponds to the largest Hartmann number. The application of a transverse magnetic field to the electrically conducting polymer flow clearly counteracts the primary flow. Fig. 4 however reveals that secondary (cross flow i.e. transverse) shear stress is conversely enhanced with greater magnetic parameter. This is invoked due to the Hall current which generates a secondary (cross) flow in the boundary layer regime. Therefore, a lateral acceleration will also be induced in the cross flow with increasing magnetic body force parameter. It is also noteworthy that both finite difference and finite element methods remain very popular and versatile for electromagnetic mechanics and other applications including sensors, power transformers, electrical machines, antennas etc. Representative studies include Zhao et al. [43] on magnetic thrust bearings, NasiriGheidari [44] on linear resolvers in electrical energy conversion, Moradnouri et al. [45] on genetic simulation of transformer flux diverters, Borghi and Cristofolini [46] on finite difference computation of electrodynamics in MHD energy generation systems and Yoshida et al. [47] on finite element and finite difference open cycle hydromagnetic energy generators.

\section{RESULTS AND DISCUSSION}

Extensive computations have been performed for the present problem and are depicted in Figs. 544. These figures illustrate the influence suction parameter $(\varepsilon)$, magnetic parameter $(M)$, Hall current $\left(\beta_{e}\right)$, Prandtl number $\left(P_{r}\right)$, Schmidt number $\left(S_{c}\right)$, microrotation parameter $(\Delta)$, vortex viscosity $(\lambda)$, spin gradient viscosity $(\Lambda)$, thermal Grashof number $\left(G_{r}\right)$, species Grashof number $\left(G{ }_{r}\right)$, ion-slip parameter $\left(\beta_{i}\right)$, Soret number $\left(S_{0}\right)$ and Eckert number $\left(E_{\mathrm{c}}\right)$. The values of Grashof number, $G_{r}$ and modified Grashof number, $G r^{*}$ for the heat transfer are taken to be positive. Since the most important fluids are atmospheric air, salt water and water, the results are limited to $\operatorname{Pr}=$ 0.71 (Prandtl number for air at 20 Celsius), $P r=1.0$ (Prandtl number for electrolytic solution such as salt water at 20 Celsius), $\operatorname{Pr}=7.0$ (Prandtl number corresponding to water at 20 Celsius). For Schmidt number, $S c$, values selected are as follows: $S_{c}=0.6$ for water vapour diffusing in air, 0.95 for carbon dioxide diffusing in low density materials and 5.0 for denser molecular weight gases in highly viscous fluid [48]). Figs 5-44 depict the variations in the key engineering design parameters (wall gradients of the variables) which are presented for the primary and secondary shear stresses (primary shear stress is the gradient of primary velocity at the plate i.e. $\tau_{x}=\frac{\partial U}{\partial Y}$, and secondary shear stress is the gradient of secondary velocity, $W$, at the plate $\tau_{z}=\frac{\partial W}{\partial Y}$ at $\left.Y=0\right)$, micropolar couple stress (gradient of angular velocity, $\Omega$, at the plate i.e. $\frac{\partial \Omega}{\partial Y}$ at $Y=0$ ), Nusselt number (gradient of 
temperature, $\theta$, at the plate i.e. $\frac{\partial \theta}{\partial Y}$ at $Y=0$ ) and Sherwood (gradient of concentration, $\Phi$, at the plate i.e. $\frac{\partial \Phi}{\partial Y}$ at $Y=0$ ). Plots included show the variation of these functions with time $(\tau)$. The wall micro-rotation constant, $S$ is selected as 0.5 (as discussed earlier), representing weak microelement concentrations. To achieve "steady-state solutions" for the computations, these are executed up to a dimensionless time of $\tau=80.99$. It has been verified that computational values exhibit very little variation after $\tau=10$. Thus, the solution attained at $\tau=20$ is essentially the steady state solution. Hence the primary (longitudinal) velocity, transverse (secondary i.e. crossflow) velocity, angular velocity, temperature and concentration profiles are visualized at three key time stages $\tau=1,7,20$.

Figs 5-44 illustrate the time development of the dimensionless steady-state shear stresses (namely $\left.\tau_{\chi} \tau_{z}\right)$, couple stress $\left(M_{c}\right)$, Nusselt number $(N u)$ and Sherwood number $(S h)$ versus dimensionless time $(\tau)$.

Fig. 5 depicts the effect of magnetic parameter (Hartmann number, $M$ ) on the steady-state couple stress. Micropolar couple stress is slightly increased initially with increasing magnetic field. At low time values, in fact negative spin of the micro-elements is induced. However, with greater progression in time, couple stress is observed to increase steadily and peaks at very high time values. For the majority of time values studied, the couple stress is sustained as positive. A significant reduction in couple stress accompanies increasing values of the magnetic parameter as time progresses. Spin of micro-elements is therefore inhibited with increasing time. This has implications in materials processing since the gyratory motion of microelements (suspended particles) is damped as time elapses which in turn interacts with the velocity fields and leads to modifications in shear stress distributions. Fig. 6 indicates that secondary shear stress $\left(\tau_{z}\right)$ is elevated markedly with an increase in Hall parameter, $\left(\beta_{\mathrm{e}}\right) . \tau_{\mathrm{z}}$ generally also increases steadily from the initiation of flow but tends to plateau after a short elapse of time. Thereafter time is found to have no effect on the secondary shear stress. Furthermore, computations (not shown for brevity) have revealed that the primary shear stress, $\tau_{x}$, is not affected by the Hall parameter, as anticipated, since this parameter is associated with the cross flow (secondary flow field). These trends have also been reported by Datta and Mazumder [25] and Ram [26], even for Newtonian flows. It has also been reported in other studies including Bég et al. [24] and Zueco et al. [32] and Cramer and Pai [33]. The trends in fig. 6 confirm that an electrically-conducting fluid is strongly affected by Hall currents in the presence of transverse magnetic field. The effects of Hall current gives rise to the Lorentzian force in the z-direction. Therefore, a crossflow is generated in the lateral direction. This aids in the secondary flow development and accelerates the secondary flow, 
manifesting in an enhancement in secondary shear stress $\left(\tau_{z}\right)$ with increasing values of $\beta_{\mathrm{e}}$, even for relatively low values of $\beta_{\mathrm{e}}$.

Figs. 7-11 illustrate the time development in primary and secondary shear stresses, couple stress, Nusselt number and Sherwood number for different values of dimensionless Prandtl number, $P_{r}$. Prandtl number, $P_{r}$, defines the ratio of momentum diffusivity to the thermal diffusivity. It is evident from Figs. 7 and 8, that both shear stress components, $\tau_{x}, \tau_{z}$ decrease as increases. Initially as time is increased the shear stress components both increase; however, after a short elapse in time they attain a maximum value and remain invariant with time thereafter. Couple stress (fig. 9) is observed to be initially increased slightly with rising Prandtl number but decreases initially with time. However, with greater elapse of time, there is a strong increase in wall couple stress and also a decrease with Prandtl number. Figs.10 and 11 demonstrate that with increasing values of $P_{r}$, steady state Nusselt number increases significantly whereas Sherwood number decreases. Heat transfer rate at the wall is therefore elevated whereas species diffusion (mass transfer) rate is depressed with greater Prandtl number. With an increase in time, both Nusselt and Sherwood numbers are observed to decrease initially and then sustain constant values for subsequent increase in time.

Figs 12-16 illustrate the temporal evolution of the flow characteristics for different values of Schmidt number, $S_{c}$. Schmidt number embodies the ratio of the momentum diffusivity to the mass (species) diffusivity. It physically relates the relative thickness of the hydrodynamic boundary layer and mass transfer (species concentration) boundary layer. Evidently both steady-state primary and secondary shear stress components, $\tau_{x,} \tau_{z}$ (figs. 12,13) decrease with increasing Schmidt number, although the primary shear stress magnitudes are several orders of magnitude higher. With an increase in time, both shear stress components initially rise sharply and then attain a constant value which remains unchanged with subsequent times. Fig.14 indicates that wall couple stress initially increases with Schmidt number and time, $\tau$, but thereafter with greater passage of time, couple stress is strongly depressed with increasing Schmidt number. Nusselt number and Sherwood number (Figs. 15 and 16) are both markedly enhanced with an increase in Schmidt number. Heat and mass transfer rates at the wall are therefore greater for higher density species diffusing, as opposed to lower molecular weight species. The boost in Nusselt and Sherwood numbers implies a concomitant decrease in temperatures and species concentration values within the body of micropolar fluid.

Fig. 17 illustrates the time development of couple stress for different values of Eringen vortex viscosity parameter, $\lambda$. Couple stress decreases as $\lambda$ rises, indicating that as viscosity is enhanced, the rotary motions of micro-elements are depressed, and local spinning is suppressed. For lower values of this vortex viscosity parameter, the couple stress increases with time to a steady-state value. However, 
for larger values, there is an initial very steep decay in couple stress, following which the couple stress begins to ascend towards the steady-state value.

Fig. 18 illustrates the evolution of couple stress with $\Lambda$ i.e. the spin gradient viscosity parameter (= $\left.\frac{\gamma}{\rho j \vartheta}\right)$. Initially there is a decrease in spin reversal in micro-elements leading to lesser negative wall couple stress values with increasing spin-gradient viscosity, at low times. With further elapse in time, the wall couple stress is observed to be significantly depressed with increasing spin gradient parameter and steady-state values are achieved relatively smoothly. Spin gradient viscosity is therefore a critical micropolar parameter influencing the angular velocity gradient at the wall.

Figs. 19-23 depict the influence of time and thermal Grashof number (buoyancy parameter) on the micropolar flow characteristics. Primary shear stress (fig. 19) is strongly decreased since thermal buoyancy opposes primary momentum development. However, this is compensated for by the enhancement in secondary shear stress (fig. 20) with increasing Grashof numbers $\left(G_{r}\right)$. Magnitudes of primary shear stress, as noted earlier, are however considerably greater than secondary shear stress, at any time and for any thermal Grashof number. Buoyancy force acts like a reverse pressure gradient which decelerates the primary flow whereas it accentuates the secondary flow. With increasing thermal Grashof number, couple stress is initially depressed at low values of time; however, it is significantly boosted with thermal Grashof number at larger time values. Figs. 22 and 23 show that Nusselt number is depressed with increasing $G_{r}$ values whereas the Sherwood number is accentuated. Mmass transfer at the wall is therefore aided with greater buoyancy (larger Grashof number), whereas heat transfer is inhibited.

Figs. 24-28 illustrate the response of the flow characteristics with species Grashof number $\left(G_{r}{ }^{*}\right)$ and time. Species buoyancy force defines the ratio of the species (solutal) buoyancy force to the viscous hydrodynamic force. For $G_{r}{ }^{*}=1$ both forces contribute equally. Both primary and secondary shear stress components are strongly elevated with increasing species Grashof number (figs. 24, 25). Couple stress (fig. 26) is initially decreased with greater species Grashof number at low times, whereas the opposite effect is induced at higher values of time. Nusselt number is observed to be very considerably decreased with increasing thermal Grashof number (fig. 27), whereas the Sherwood number demonstrates the opposite behaviour i.e. Sherwood number is elevated with greater species Grashof numbers, indicating that mass diffusion to the plate is aided with greater species buoyancy forces. Thermal diffusion to the plate is however opposed with greater species buoyancy forces. 
Figs. 29-32 present the distribution of shear stress components, Nusselt number and Sherwood number, with time for various Eckert numbers $\left(E_{c}\right)$. Eckert number arises owing to the viscous heating and also Joule heating (Ohmic dissipation) effects. It features in the last two terms of the dimensionless energy eqn. (11). The viscous heating term features quadratic terms of the primary and secondary velocity gradients, whereas the Joule heating (magnetic dissipation) term features only the quadratic terms for velocity components. $E_{c}$ is therefore expected to exert considerable influence on flow characteristics. With greater Eckert number, both primary and secondary shear stresses are both very strongly enhanced (figs. 28, 29). Conversely Nusselt number is significantly reduced with increasing Eckert number. This is due to the fact that as Eckert number increases, greater kinetic energy in the flow is transformed into thermal energy. This heats the fluid regime but draws heat away from the plate, leading to a decrease in wall heat transfer rates i.e. a reduction in Nusselt number. On the other hand, Sherwood number receives a marked enhancement with increasing Eckert number. Therefore, greater mechanical energy dissipation and conversion to heat aids in species diffusion (mass transfer) to the wall, whereas it strongly depresses heat transfer to the wall.

Figs. 33, 34 present the effect of the ionslip parameter, $\beta_{i}$, on primary and secondary shear stress components. Very little tangible influence is computed. This parameter arises in both momenta equations (8, 9) and the energy equation (11) i.e. $-\frac{M}{\left(\alpha_{e}^{2}+\beta_{e}^{2}\right)}\left(\alpha_{e} U+\beta_{e} W\right)$ in eqn. (8), $+\left(\frac{M}{\alpha_{e}^{2}+\beta_{e}^{2}}\right)\left(\beta_{e} U-\alpha_{e} W\right)$ in eqn. (9) and the modified Ohmic dissipation term, $+M \frac{E_{c}}{\left(\alpha_{e}^{2}+\beta_{e}^{2}\right)}\left(U^{2}+W^{2}\right)$ in eqn. (11), in which $\alpha_{e}=1+\beta_{i} \beta_{e}$. The impact of ion slip is vert weak, which is in stark contrast to the influence of the Hall parameter, $\beta_{e}$ as described earlier, which influences the flow characteristics quite strongly. The consideration of weak magnetic fields is no doubt a major reason for the negligible influence of ion slip parameter. This parameter $[33,34]$ is expected only to exert any influence on flow characteristics at very strong magnetic fields.

Figs. 35-39 present the effect of wall transpiration parameter $(\varepsilon)$ on the shear stress components, couple stress, Nusselt and Sherwood number plotted against transient time. We consider only the case of suction (positive $\varepsilon$ ) i.e. blowing (injection) is not considered. Clearly both shear stress components are depressed with increasing suction, since this causes the fluid to be drawn more strongly to the wall and decelerates flows. It is also interesting to note that at high values of suction, the shear stress components quickly attain steady state values, whereas at lower values $(0.5)$ there is a much more gradual ascent in both primary and secondary shear stress. Strong suction therefore while decelerating the primary and cross flows, also simultaneously stabilizes the flow regime and increases momentum boundary layer thicknesses. With increasing suction, couple stress (fig. 37) is enhanced in magnitude (values are negative for low suction indicating spin reversal of micro- 
elements). Figs 38 and 29 demonstrate that with greater suction, generally both Nusselt number and Sherwood number are significantly increased (for all time values). However, in fig. 39, we also observe that for low suction, the Sherwood number attains highest magnitudes with greater elapse of timethese magnitudes are in fact greater than those computed for higher suction values, but only for large time elapse.

Finally, figs. 40-44 present the influence of thermal-diffusion parameter (Soret number i.e. $S_{o}$ ) on flow characteristics. The Soret effect is described in the species conservation eqn. (12) by a second order temperature derivative i.e. in the term, $s_{0} \frac{\partial^{2} \theta}{\partial Y^{2}}$. Increasing Soret number generally enhances both the primary and secondary shear stress components (figs. 40,41). Couple stress is initially depressed with greater Soret number for low time values; however, with greater time values there is a strong elevation in couple stress which eventually achieves steady state values. Both Nusselt number and Sherwood number are strongly decreased with greater Soret number, as observed in figs. 43, 44 . Greater thermo-diffusive effect therefore inhibits both heat transfer and mass transfer (species diffusion) to the wall and will conversely accentuate the magnitudes of temperatures and species concentration in the boundary layer.

\section{CONCLUSIONS}

A mathematical model has been developed for transient thermosolutal magnetohydrodynamic free convection flow of an ionized incompressible viscous micropolar fluid from an infinite vertical surface in the presence of Hall current and ion-slip effects, as a model of electroconductive materials processing. The transformed nonlinear time-dependent boundary value problem has been solved computationally, subject to pertinent boundary conditions with a well-tested, stable, efficient, implicit finite difference method. Validation of the FDM solutions has been achieved with a Galerkin finite element method (FEM). The influence of Hall parameter $\beta_{e}$, lon-slip parameter $\beta_{i}$, magnetic parameter, $M$, vortex viscosity parameter $\lambda$, spin gradient viscosity parameter, $\Lambda$, Prandtl number, $P r$, Grashof number, $G_{r}$, solutal Grashof number $G_{r}{ }^{*}$, Eckert number, $E_{c}$, Schmidt number, $S_{c}$ as well as wall suction (later mass efflux) parameter, $\varepsilon$ and Soret number, $S_{o}$ on primary and secondary shear stress components, wall couple stress and Nusselt and Sherwood number, have been evaluated and presented graphically. Interesting responses in flow characteristics to these parameters have been described. The main findings are summarized below:

(i)Wall suction has a stabilizing influence on the flow regime and to enhance couple stress and both Nusselt and Sherwood numbers. 
(ii) Increasing Soret number has been shown to depress both Nusselt and Sherwood numbers whereas it enhances both primary and secondary shear stress and at larger time values boosts the couple stress.

(iii) Secondary shear stress is elevated markedly with an increase in Hall parameter and greater magnetic parameter (Hartmann number).

(iv) Micropolar couple stress is also weakly enhanced for small time values with increasing magnetic field parameter.

(V)Generally primary shear stress is enhanced with thermal Grashof number, species Grashof number, Eckert number and Soret number whereas it is suppressed with increasing Prandtl number and Schmidt number.

(VI) Secondary shear stress is observed to be enhanced with thermal Grashof number, species Grashof number, Eckert number and Soret number but depressed with increasing Prandtl number and Schmidt number.

(VII) Finally, the wall couple stress is shown to be boosted with increasing Prandtl number, Schmidt number, spin gradient viscosity parameter and Soret number but is depressed with increasing thermal Grashof number, species Grashof number, vortex viscosity and suction parameters.

The present study has neglected chemical reaction effects, which are also important in electromagnetic polymer materials processing $[49,50]$ and these will be considered in the future. Furthermore, emerging electro-conductive polymers are also featuring biological micro-organism doping for enhanced performance e.g. Zhang et al. [51] and Bhatti et al. [52] in addition to bioinspired walls (boundaries) e.g. Bhatti et al. [53]. These also constitute interesting possible pathways for extending the current study.

\section{REFERENCES}

[1] S. Way., Electromagnetic propulsion for cargo submarines, AIAA J. Hydronautics, 2, 49-57 (1968).

[2] E.M. Braun, F.K. Lu, D.R. Wilson, Experimental research in aerodynamic control with electric and electromagnetic fields, Progress in Aerospace Sciences, 45, 30-49 (2009).

[3] M. Ferdows, M. S. Khan and O. Anwar Bég, Numerical study of transient magnetohydrodynamic radiative free convection nanofluid flow from a stretching permeable surface, Proc. IMechE-Part E: J. Process Mechanical Engineering, 228 (3) 1-25 (2014). 
[4] V.N. Selyanenkov, Formation of the weld pool in a longitudinal magnetic field in argon-arc welding. Welding Production, 22, 50-53 (1975).

[5] M.J.Uddin, M. Ferdows and O. Anwar Bég, Group analysis and numerical computation of magneto-convective non-Newtonian nanofluid slip flow from a permeable stretching sheet. Applied Nanoscience, 4, 897-910(2014).

[6] O. Anwar Bég, J. Zueco and L. M. López-Ochoa, Network numerical analysis of optically thick hydromagnetic slip flow from a porous spinning disk with radiation flux, variable thermophysical properties and surface injection effects, Chemical Engineering Communications, 198, 3, 360-384 (2011).

[7] I. Nliuta and F. Larachi, Magnetohydrodynamics of trickle bed reactors: Mechanistic model, experimental validation and simulations, Chemical Engineering Science, 58, 297-307 (2003).

[8] D. Tripathi, O. Anwar Bég and S.K. Pandey, Transient magneto-peristaltic flow of couple stress biofluids: a magneto-hydro-dynamical study on digestive transport phenomena, Mathematical Biosciences, 246, 72-83 (2013).

[9] O. Anwar Bég, J. Zueco, M. Norouzi, M. Davoodi, A. A. Joneidi, Assma F. Elsayed, Network and Nakamura tridiagonal computational simulation of electrically-conducting biopolymer micromorphic transport phenomena, Computers in Biology and Medicine, 44, 44-56 (2014).

[10] M.F. El-Amin, Combined effect of viscous dissipation and Joule heating on MHD forced convection over a non- isothermal horizontal cylinder embedded in a fluid-saturated porous medium, J. Magn. Magn. Materials., 263, 337- 343 (2003).

[11] M. Subhas Abel and N. Mahesha, Heat transfer in MHD viscoelastic fluid flow over a stretching sheet with variable thermal conductivity, non-uniform heat source and radiation, Applied Mathematical Modelling, 32, 1965-1983 (2008).

[12] O. Anwar Bég, S.K. Ghosh, S. Ahmed and T. A. Bég, Mathematical modelling of oscillatory magneto-convection of a couple stress biofluid in an inclined rotating channel, J. Mechanics In Medicine and Biology, 12, 1250050.1 - 1250050.35 (2012).

[13] Zheng C, Liu YQ, Zhang XX. Slip effects on MHD flow of a generalized Oldroyd-B fluid with fractional derivative. Nonlinear Anal and Real World Applicns, 13, 513-523 (2012).

[14] A. Subba Rao, V. R. Prasad, N. Bhaskar Reddy and O. Anwar Bég, On MHD flow and heat transfer of Casson rheological fluid from a horizontal circular cylinder with partial slip, National Conf. Innovations in Mechanical Engineering, MITS, Madanapelle, India, June (2013).

[15] A. C. Eringen, Theory of micropolar fluids, J. Math. Mech. 16, 1-18 (1966).

[16] A. C. Eringen, Microcontinuum Field Theories-II: Fluent Media, Springer-Verlag, New York, Inc. (2001). 
[17] O Anwar Bég, J. Zueco and T.B. Chang, Numerical analysis of hydromagnetic gravity-driven thin film micropolar flow along an inclined plane, Chemical Engineering Communications, 198, 312- 331 (2010).

[18] N. T. Eldabe, E. F. Elshehawey, E. M. E. Elbarbary and N. S. Elgazery, Chebyshev finite difference method for MHD flow of a micropolar fluid past a stretching sheet with heat transfer, Appl. Math. Comput. 160, 437-450 (2005).

[19] O. Anwar Bég, M.M. Rashidi, T. A. Bég and M. Asadi, Homotopy analysis of transient magnetobio-fluid dynamics of micropolar squeeze film: a model for magneto-bio-rheological lubrication, J. Mechanics In Medicine and Biology, 12, 1250051.1 - 1250051.21 (2012).

[20] S. Rawat, R. Bhargava, Renu Bhargava and O. Anwar Bég, Transient magneto-micropolar free convection heat and mass transfer through a non-Darcy porous medium channel with variable thermal conductivity and heat source effects, Proc. I Mech E Part C- J. Mechanical Engineering Science, 223, 2341-2355 (2009).

[21] T. Hayat, S. A. Shehzad and M. Qasim, Mixed convection flow of a micropolar fluid with radiation and chemical reaction, Int. J. Numer. Methods Fluids, 67, 1418-1436 (2011).

[22] L. Kumar, Finite element analysis of combined heat and mass transfer in hydromagnetic micropolar flow along a stretching sheet, Comput. Mater. Sci. 46, 841-848 (2009).

[23] Haque, M. Z. and Alam, M. M., Micropolar fluid behaviour effects on unsteady MHD heat and mass transfer flow with constant heat and mass fluxes, Joule heating and viscous dissipation, A. M. S. E. (Series B Mechanics and Thermics), 82 (2), 1-25 (2011).

[24] O. Anwar Bég OA, Zueco J, Takhar HS. Unsteady magnetohydrodynamic Hartmann-Couette flow and heat transfer in a Darcian channel with Hall current, ionslip, viscous and Joule heating effects: network numerical solutions, Commun Nonlinear Sci Numer Simul., 14, 1082-97 (2009).

[25] N. Datta and B.S. Mazumder, Hall effects on hydromagnetic free convection past an infinite porous flat plate, J. Math. Phys. Sci., 10, 59-67 (1976).

[26] P.C. Ram, Hall effects on the hydromagnetic free convective flow and mass transfer through a porous medium bounded by an infinite vertical porous plate with constant heat flux, Int. J. Energy Res., 12, 227-234 (1988).

[27] A. Raptis and N.G. Kafoussias, Hydro-magnetic free convection and mass transfer flow with Hall currents, Can. J. Phys., 60, 1275-1280 (1982).

[28] M.A. Sattar and M.M. Hussain, Unsteady hydromagnetic free convection flow with hall current and mass transfer along an accelerated porous plate with time dependent temperature and concentration, Can. J. Phys., 70, 369-374 (1992). 
[29] O. Anwar Bég, Lik Sim, J. Zueco and R. Bhargava, Numerical study of magnetohydrodynamic viscous plasma flow in rotating porous media with Hall currents and inclined magnetic field influence, Communications in Nonlinear Science and Numerical Simulation, 15, 345-359 (2010).

[30] J. Zueco, O. Anwar Bég and L.M. Lopez-Ochoa, Non-linear transient hydromagnetic partially ionised dissipative Couette flow in a non-Darcian porous medium channel with Hall, ion slip and Joule heating effects, Progress in Computational Fluid Dynamics, 11, 2, 116-129 (2011).

[31] S K. Ghosh, O. Anwar Bég and A. Aziz, A mathematical model for magnetohydrodynamic convection flow in a rotating horizontal channel with inclined magnetic field, magnetic induction and Hall current effects, World J. Mechanics, 1 (3), 137-154 (2011).

[32] J. Zueco, P. Eguía, E. Granada, J. L. Míguez, O. Anwar Bég, An electrical network for the numerical solution of transient MHD Couette flow of a dusty fluid: Effects of variable properties and Hall current, Int. Comm. Heat Mass Transfer, 37, 1432-1439 (2010).

[33] Cramer KC, Pai S-I. Magnetofluid Dynamics For Engineers and Applied Physicists, MacGraw-Hill, USA (1973).

[34] O. Anwar Bég, S.K. Ghosh and Tasveer A. Bég, Applied Magnetofluid Dynamics: Modelling and Computation, 442 pages, Lambert Academic, Saarbrucken, Germany (2011).

[35] S. K. Ghosh, O. Anwar Bég and M. Narahari, Hall effects on MHD flow in a rotating system with heat transfer characteristics, Meccanica, 44, 741-765 (2009).

[36] O. Anwar Bég, Numerical methods for multi-physical magnetohydrodynamics, Chapter 1, pp. 1-110, New Developments in Hydrodynamics Research, Nova Science, New York, September (2012).

[37] O. Anwar Bég, M. Ferdows, Explicit numerical simulation of magnetohydrodynamic nanofluid flow from an exponential stretching sheet in porous media, Applied Nanoscience (2013). (15 pages) DOI 10.1007/s13204-013-0275-0

[38] Md Mainul Hoque; Md Mahmud Alam; M Ferdows, O. Anwar Bég, Numerical simulation of Dean number and curvature effects on magneto-biofluid flow through a curved conduit, Proc. IMECHE- Part H; J. Engineering in Medicine (2013). DOI: 10.1177/0954411913493844

[39] D. Gupta, Lokendra Kumar, O. Anwar Bég and Bani Singh, Finite element simulation of mixed convection flow of micropolar fluid over a shrinking sheet with thermal radiation, Proc IMechEPart E: J. Process Mechanical Engineering, 228 (1) 61-72 (2014).

[40] M.D. Shamshuddin, S.R. Mishra, O. Anwar Bég and A. Kadir, Unsteady reactive magnetic radiative micropolar flow, heat and mass transfer from an inclined plate with Joule heating: a model for magnetic polymer processing, Proc. IMechE- Part C. - Mechanical Engineering Science, 223, 4, 1-16 (2019). 
[41] M.D. Shamshuddin, O. Anwar Bég, Siva Reddy Sheri and A. Kadir, Rotating unsteady multiphysico-chemical magneto-micropolar transport in porous media: Galerkin finite element study, Computational Thermal Sciences, 10(2):167-197 (2018).

[42] Shamshuddin MD, Siva Reddy Sheri and O Anwar Bég, Oscillatory dissipative conjugate heat and mass transfer in chemically reacting micropolar flow with wall couple stress: A finite element numerical study, Proc IMechE Part E: J Process Mechanical Engineering, 233 (1), 4864 (2019).

[43] X. Zhao, C. Shuai, W. X, D. Chen and Z. Hu, "Force and Stiffness Calculation and Optimization of Permanent Magnetic Thrust Bearing Used in Vessels," International Journal of Applied Electromagnetics and Mechanics, Pre-press, pp. 1-16, 2020.

[44] Z. Nasiri-Gheidari, "Design, Performance Analysis, and Prototyping of Linear Resolvers," IEEE Trans. Energy Conversion, vol. 32, no. 4, pp. 1376-1385, Dec. 2017.

[45] Moradnouri, M. Vakilian, A. Hekmati and M. Fardmanesh, "Optimal Design of Flux Diverter Using Genetic Algorithm for Axial Short Circuit Force Reduction in HTS Transformers," IEEE Trans. Appl. Supercond., vol. 30, no. 1, pp. 1-8, Jan. 2020.

[46] Borghi, C A; Cristofolini, A, Analysis of the electrodynamics in an MHD generator channel, $33^{R D}$ Symposium on engineering aspects of magnetohydrodynamics, Tullahoma, TN (United States), 13-15 June (1995).

[47] M.Yoshida, J.Umoto, K. Komaya, Applications of equivalent circuit method to threedimensional analysis of open-cycle diagonal type MHD generator, Energy Conversion and Management, Volume 21, Issue 3, 1981, Pages 219-228.

[48] C.D. Han, Rheology and Processing of Polymeric Materials, Oxford University Press, USA (2007).

[49] Rashid Mehmood, S. Rana, O. Anwar Bég and A. Kadir, Numerical study of chemical reaction effects in magnetohydrodynamic Oldroyd-B oblique stagnation flow with a non-Fourier heat flux model, J. Brazilian Soc. Mech Sci. Eng. (2018). https://doi.org/10.1007/s40430-018-14464 (14 pages)

[50] N. Shukla, P. Rana, O. Anwar Bég, A. Kadir and Bani Singh, Unsteady electromagnetic radiative nanofluid stagnation-point flow from a stretching sheet with chemically reactive nanoparticles, Stefan blowing effect and entropy generation, Proc. IMechE: Part N-Journal of Nanomaterials, Nanoengineering and Nanosystems (2018). DOI: 10.1177/2397791418782030 (14 pages)

[51] L. Zhang, M. B. Arain, M. M. Bhatti, A. Zeeshan \& H. Hal-Sulami, Effects of magnetic Reynolds number on swimming of gyrotactic microorganisms between rotating circular plates filled with nanofluids. Applied Mathematics and Mechanics (English Edition), 41(4), 637-654 (2020). 
[52] M.M. Bhatti,; Shahid, A.; Abbas, T.; Alamri, S.Z.; Ellahi, R., Study of activation energy on the movement of gyrotactic microorganism in a magnetized nanofluids past a porous plate. Processes, 8(3), p.328 (2020).

[53] M. M. Bhatti, Rahmat Ellahi, A. Zeeshan, M. Marin and N. Ijaz, Numerical study of heat transfer and Hall current impact on peristaltic propulsion of particle-fluid suspension with compliant wall properties. Modern Physics Letters B, Vol. 33, No. 35, 1950439 (2019). 


\section{FIGURES AND CAPTIONS}

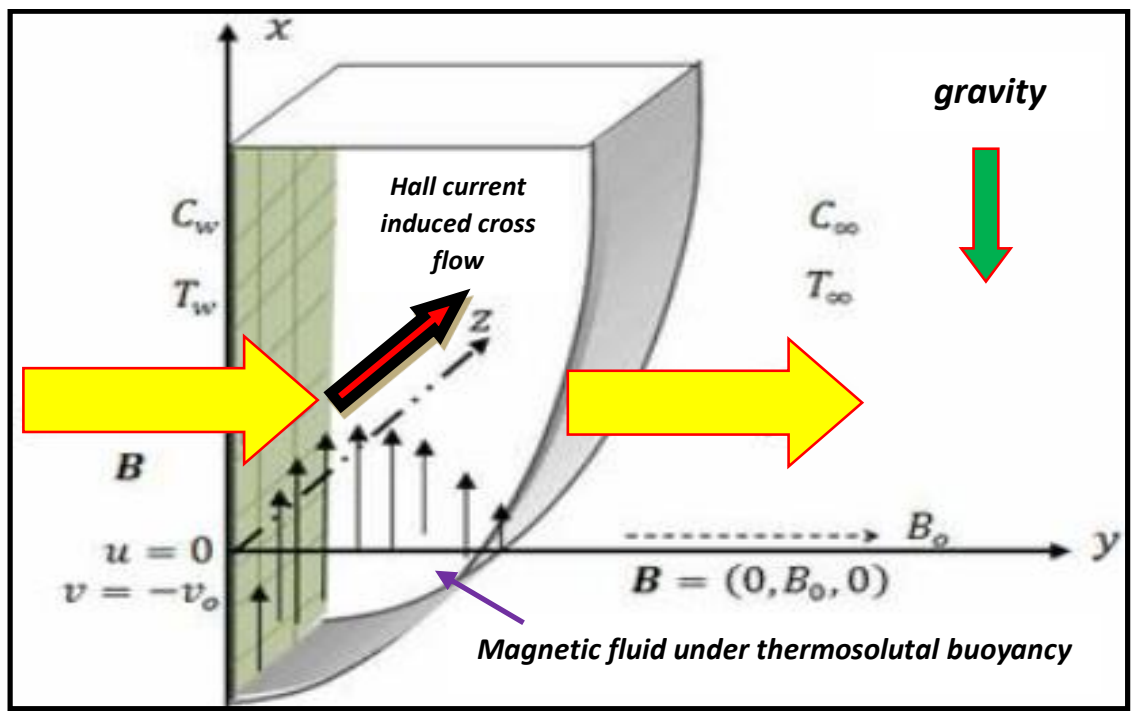

Fig. 1: Magnetohydrodynamic micropolar materials processing flow system

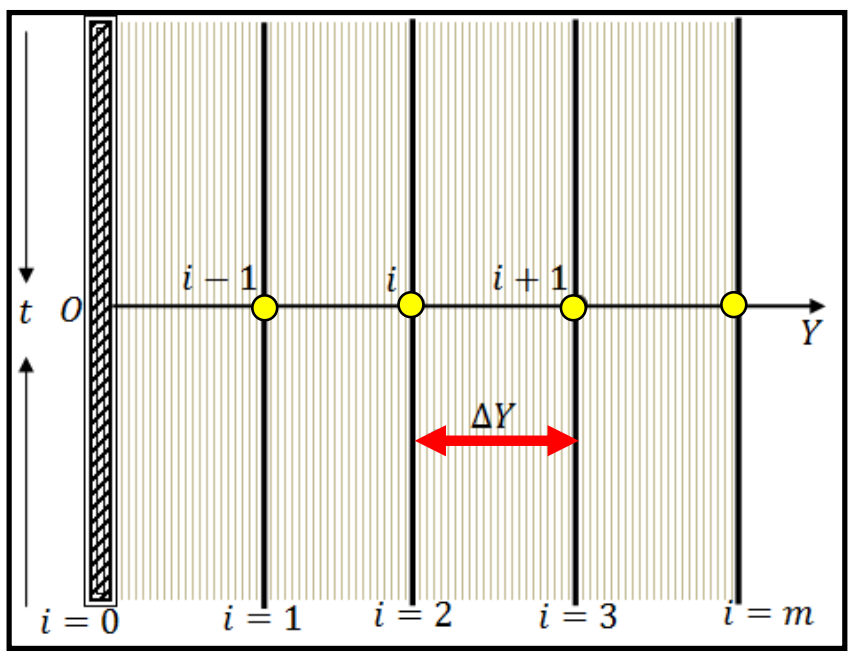

Fig 2: Finite difference numerical grid (mesh) 


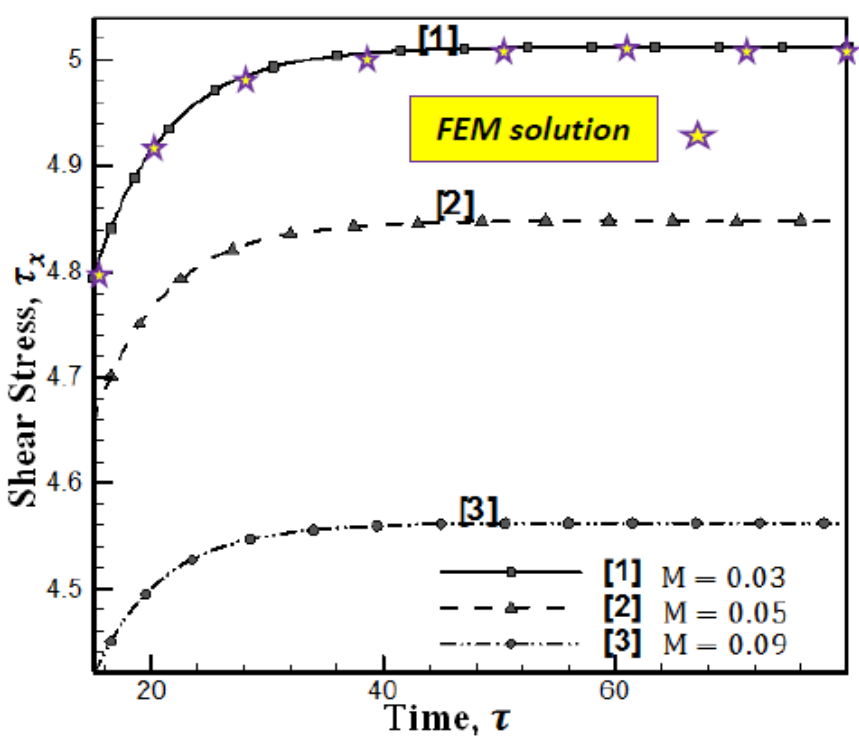

Fig. 3. Comparison of primary shear stress $\tau_{x}$ for different values of $M$ with FDM and FEM.

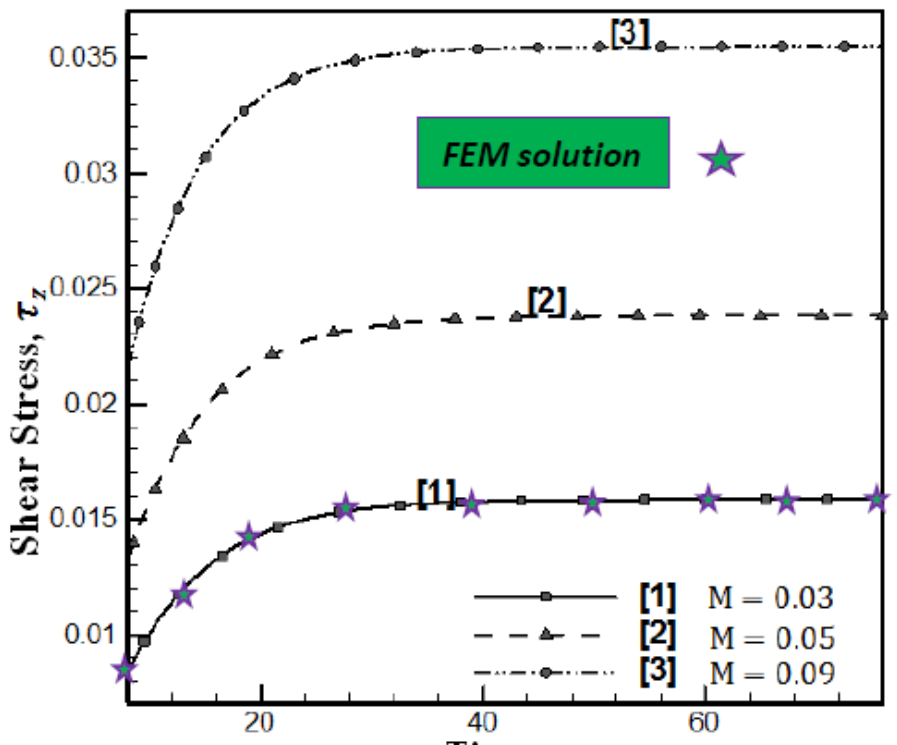

Time, $t$

Fig. 4. Comparison of secondary shear stress $\tau_{z}$ for different values of $M$ with FDM and FEM. 


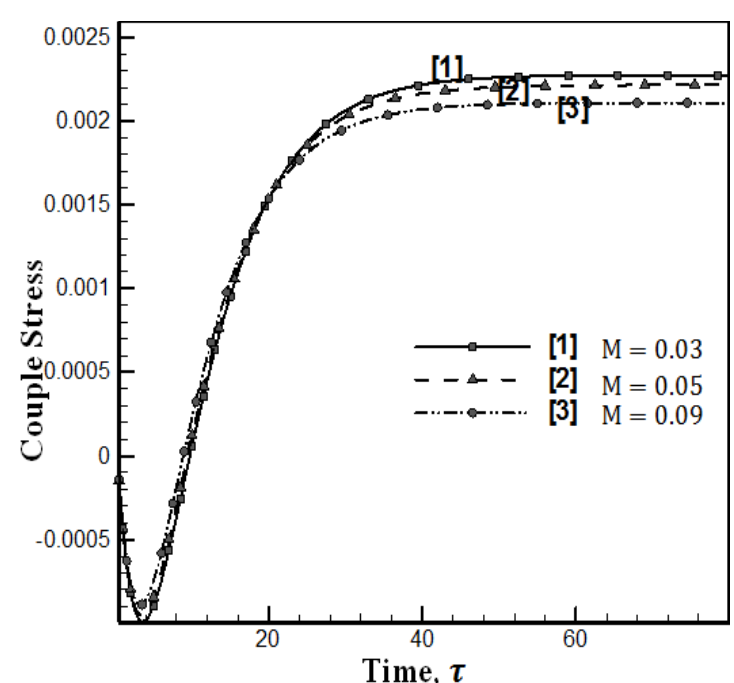

Fig. 5. Couple stress for different $M$.

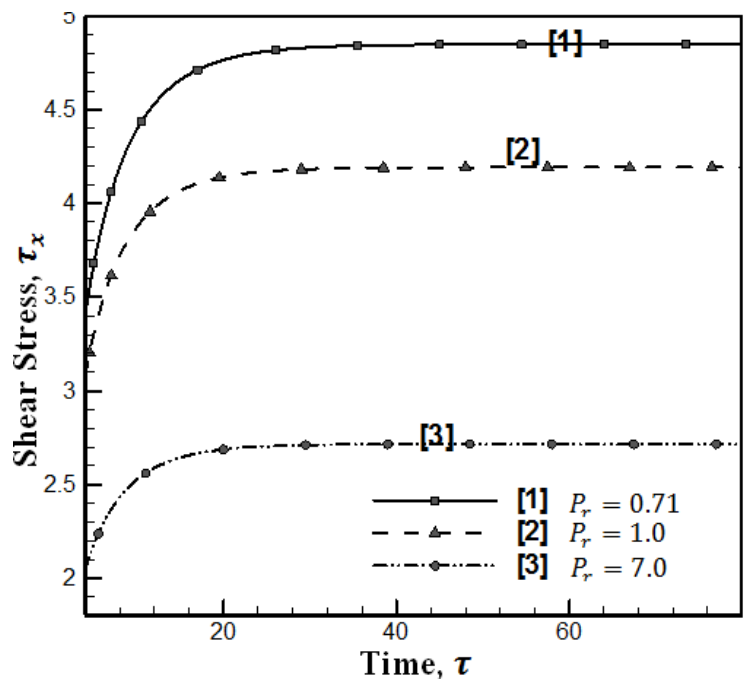

Fig. 7. Primary shear stress for different $P_{r}$

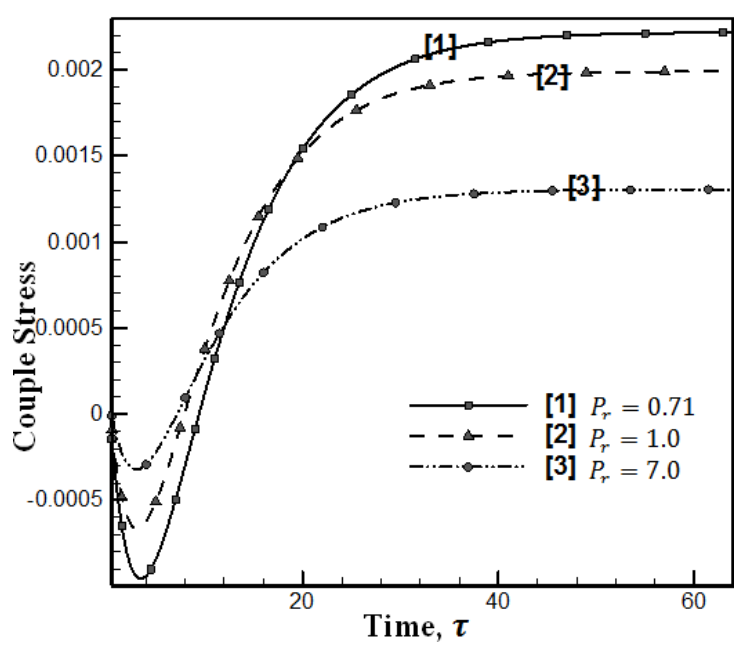

Fig. 9. Couple stress for different $P_{r}$

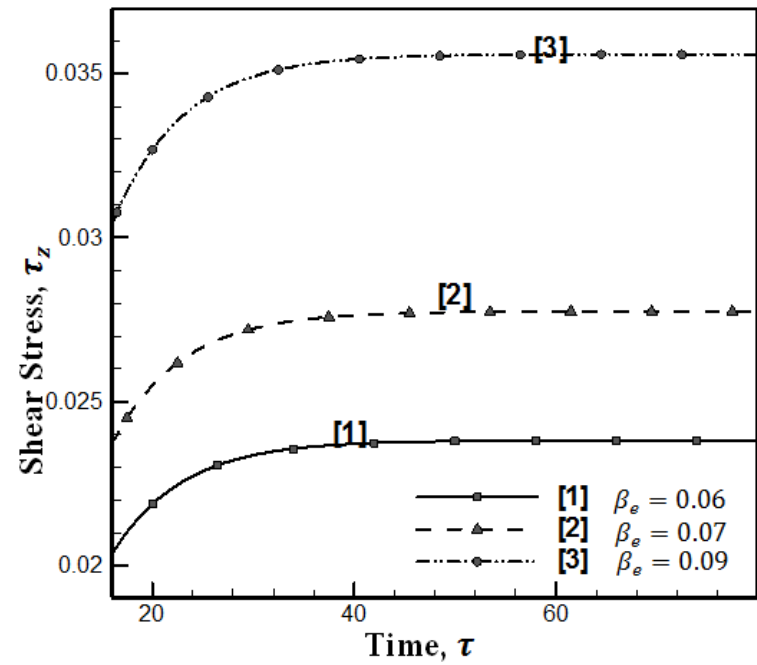

Fig. 6. Secondary shar stress for different $\beta_{e}$.

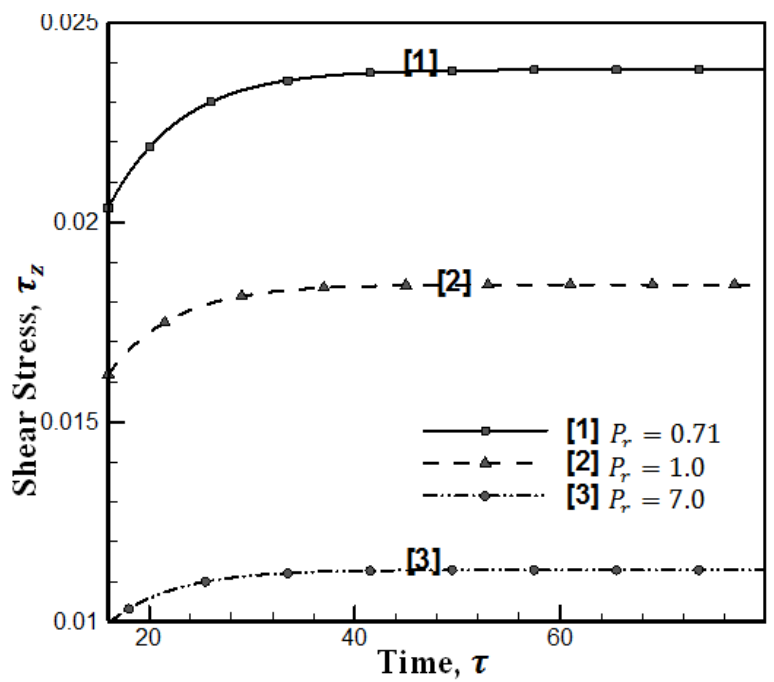

Fig. 8. Secondary shear stress for different $P_{r}$

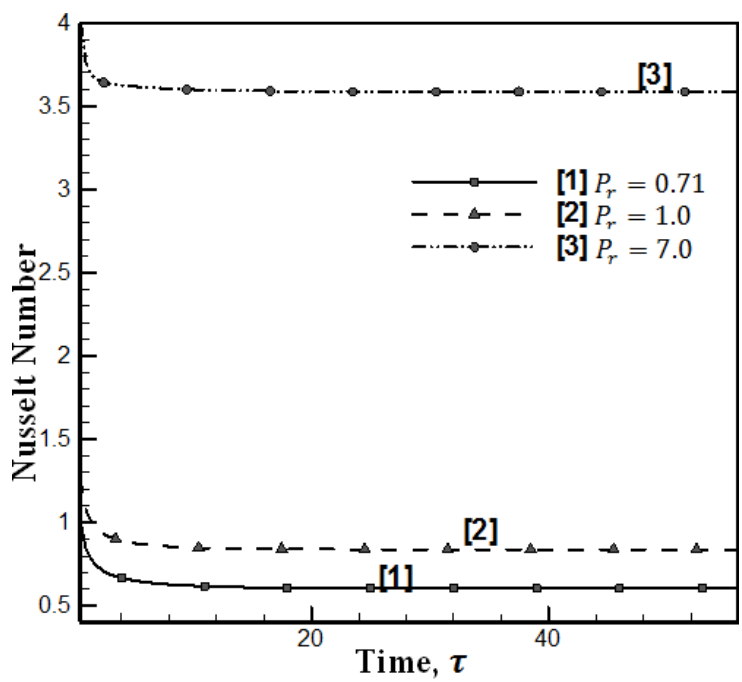

Fig. 10. Nusselt number for different $P_{r}$ 


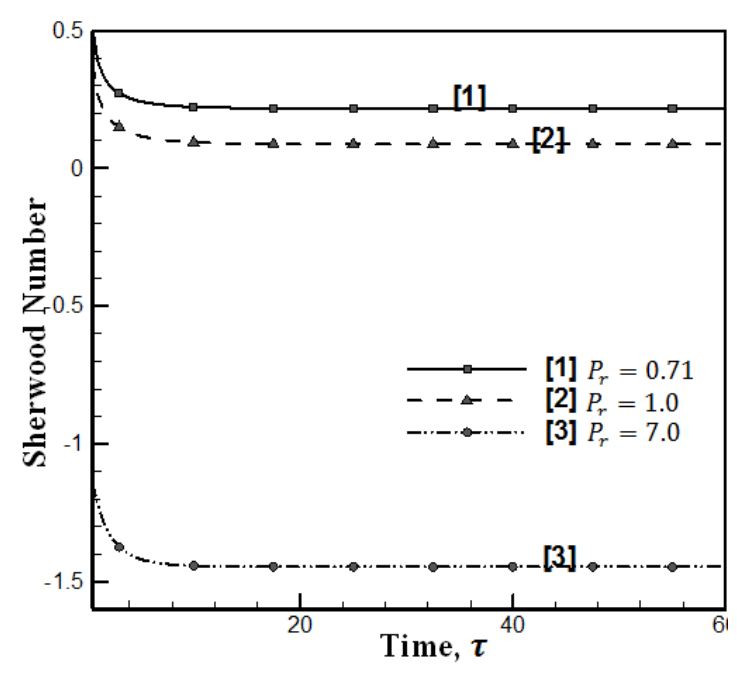

Fig. 11. Sherwood number for different $P_{r}$

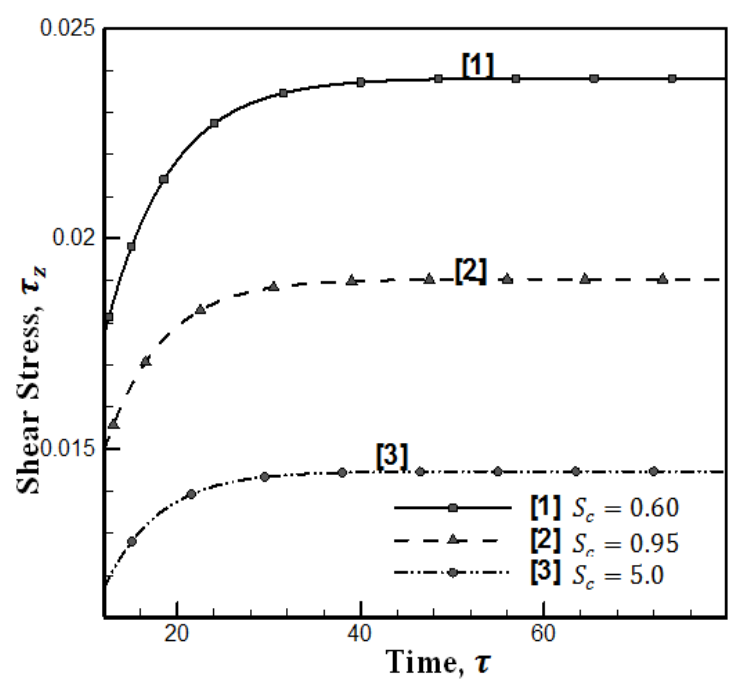

Fig. 13. Secondary shear stress for different $S_{c}$

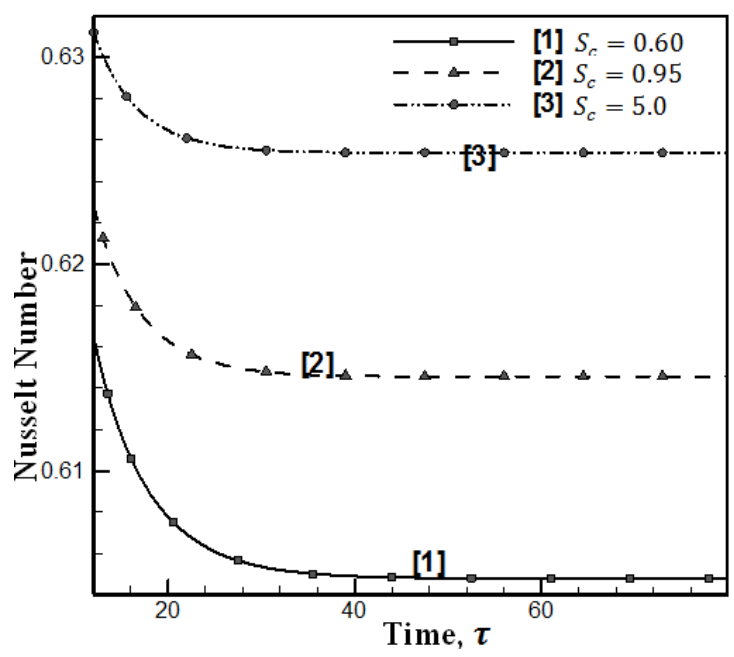

Fig. 15. Nusselt number for different $S_{c}$

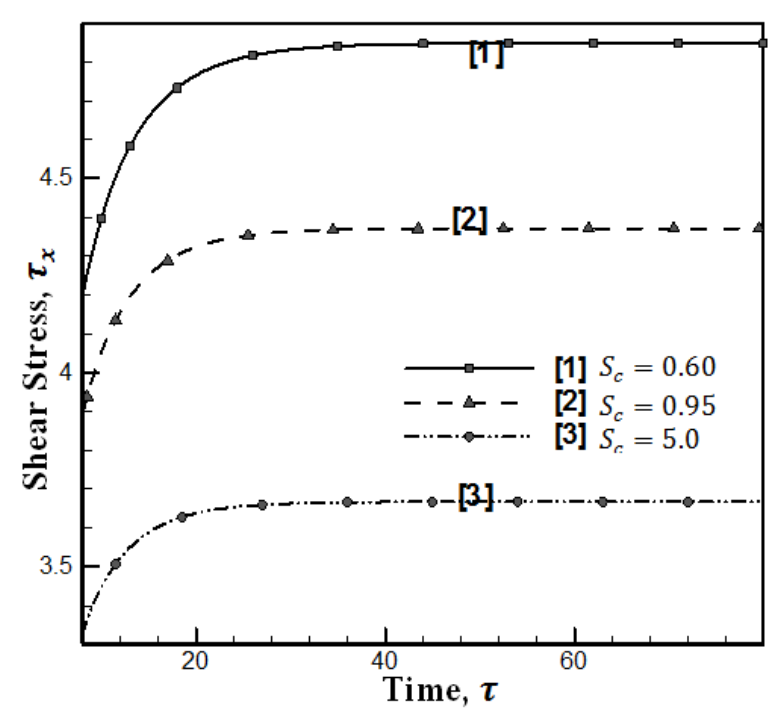

Fig. 12. Primary shear stress for different $S_{c}$

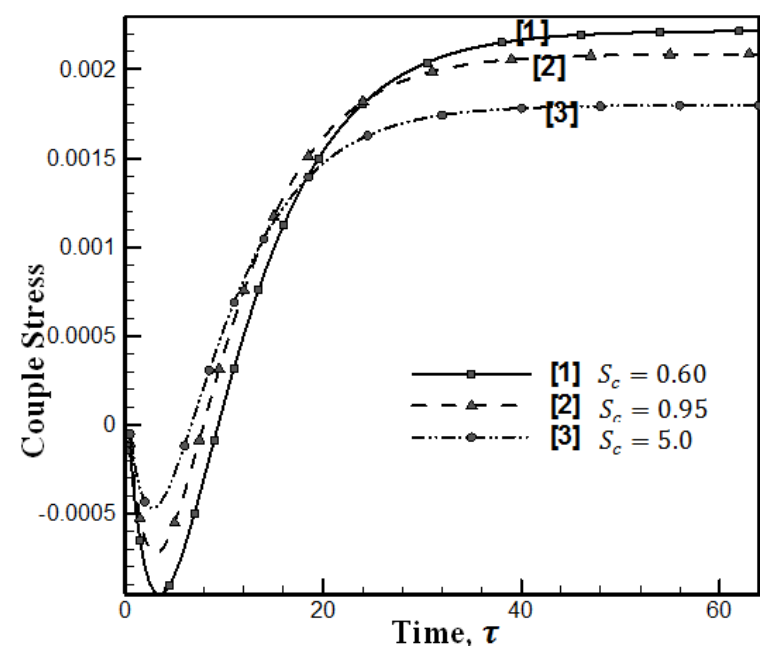

Fig. 14. Couple stress for different $S_{c}$

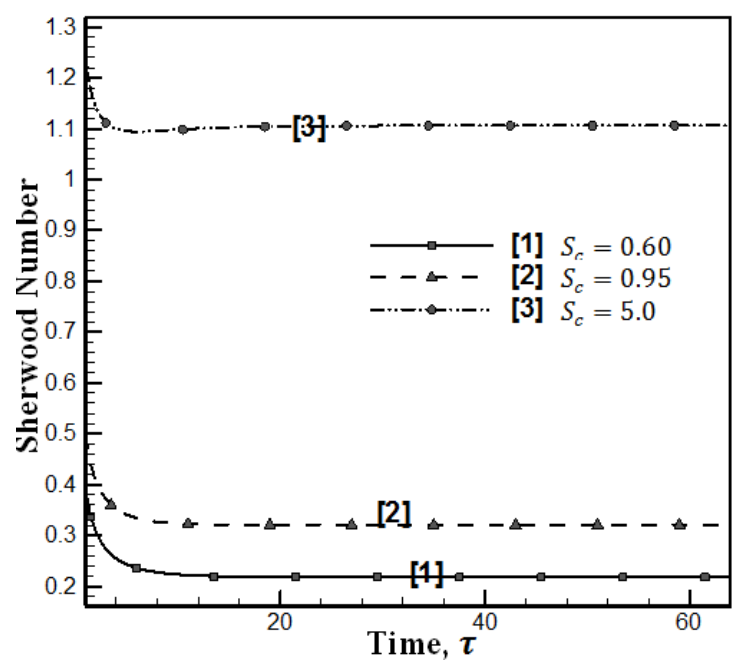

Fig. 16. Sherwood number for different $S_{c}$ 


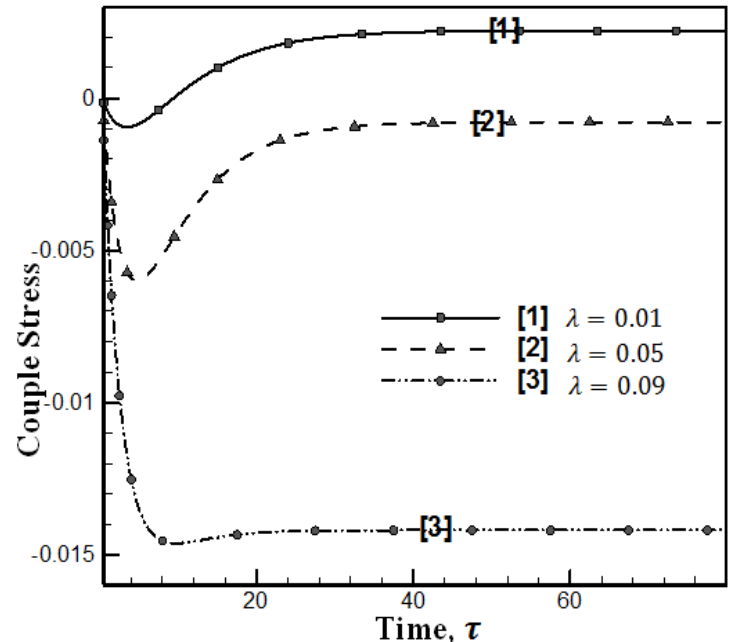

Fig. 17. Couple stress for different $\lambda$.

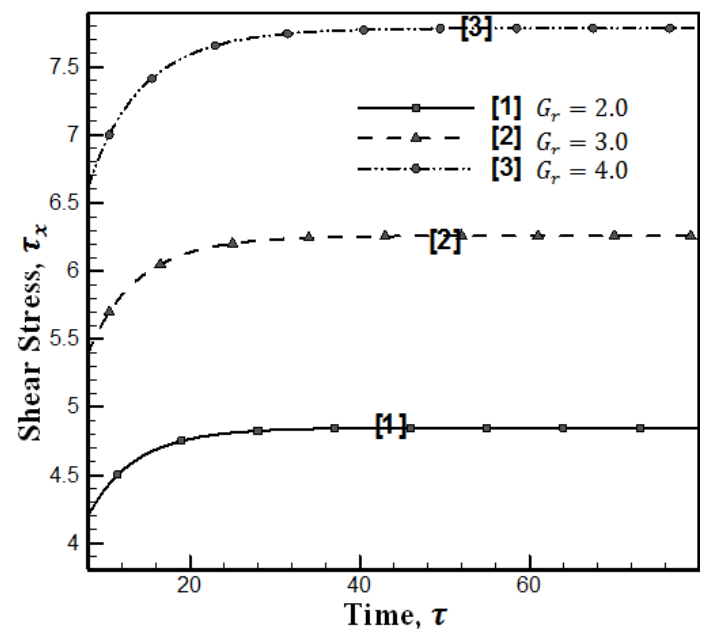

Fig. 19. Primary shear stress for different $G_{r}$.

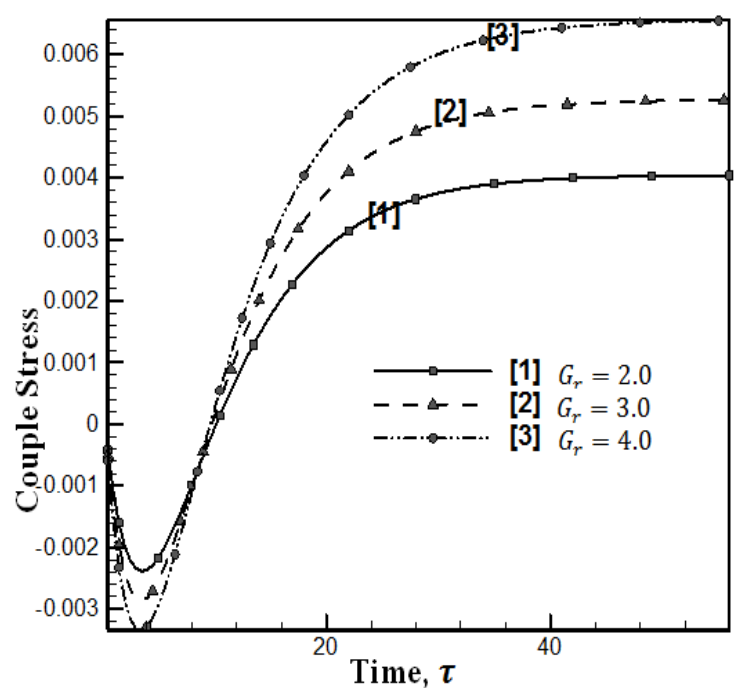

Fig. 21. Couple stress for different $G_{r}$.

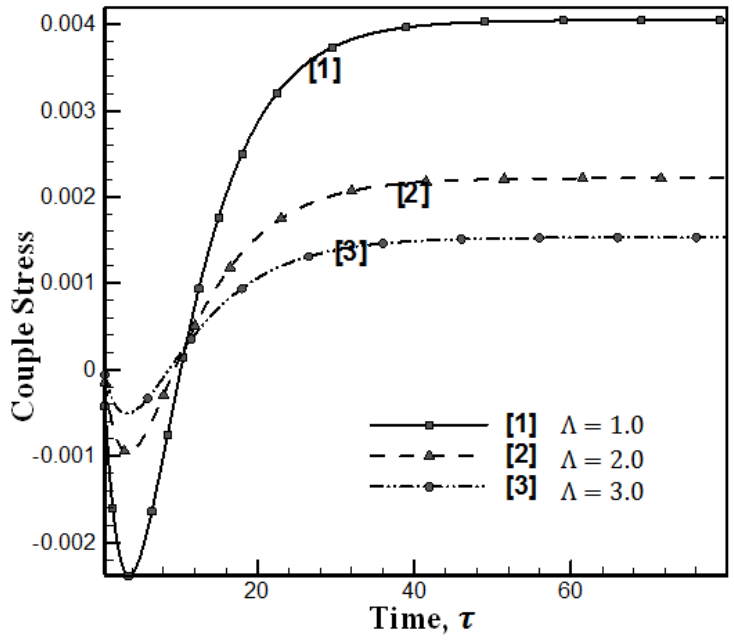

Fig. 18. Couple stress for different $\Lambda$.

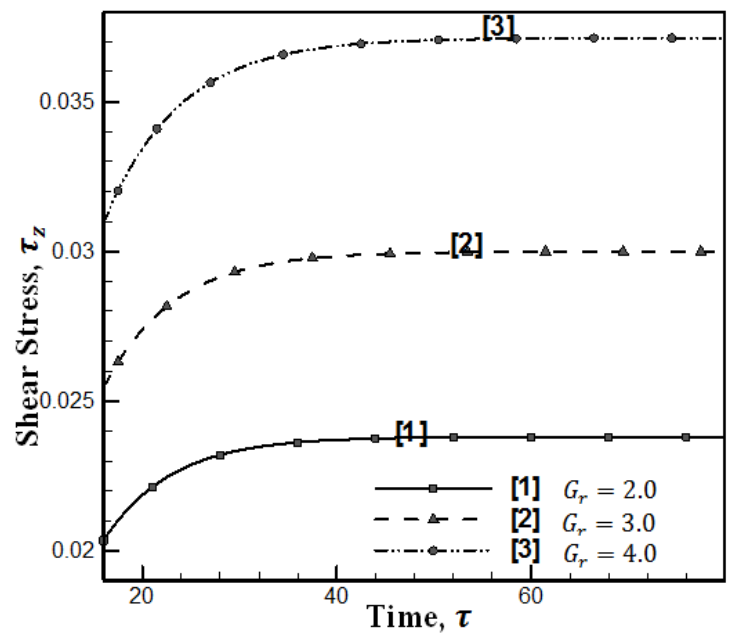

Fig. 20. Secondary shear stress for different $G_{r}$.

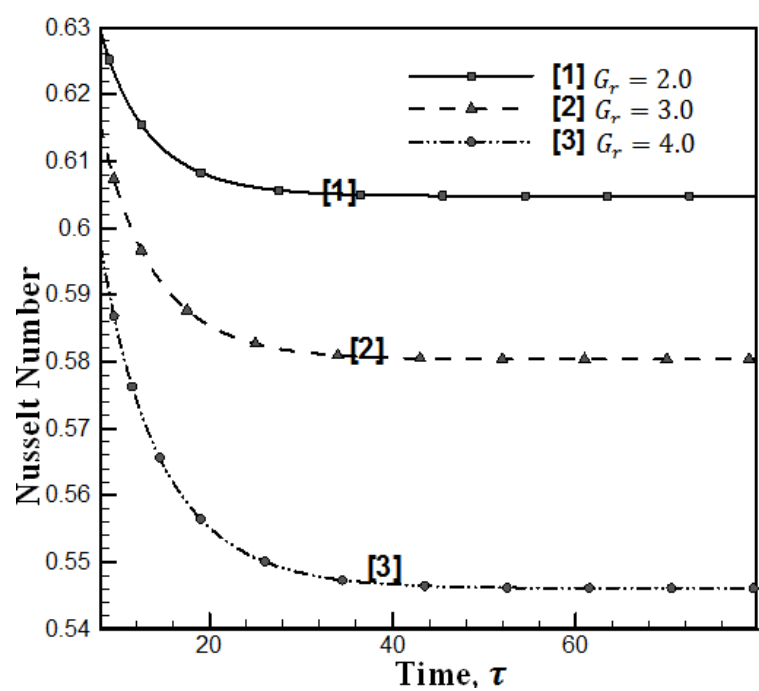

Fig. 22. Nusselt number for different $G_{r}$. 


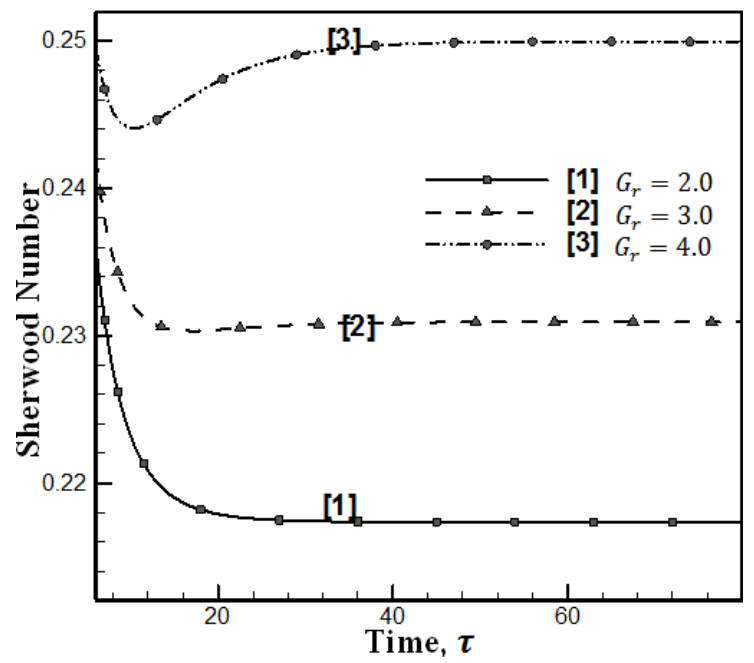

Fig. 23. Sherwood number for different $G_{r}$.

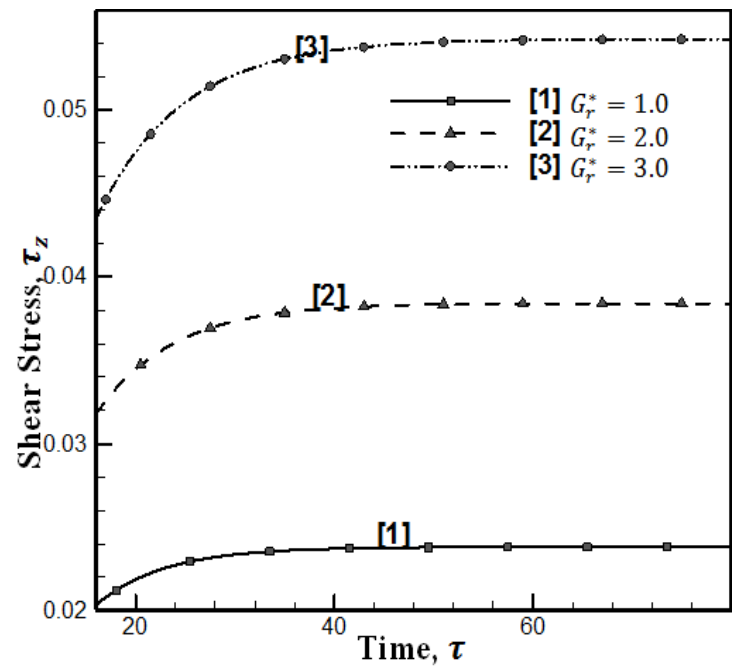

Fig. 25. Secondary stress with various $G^{*}$.

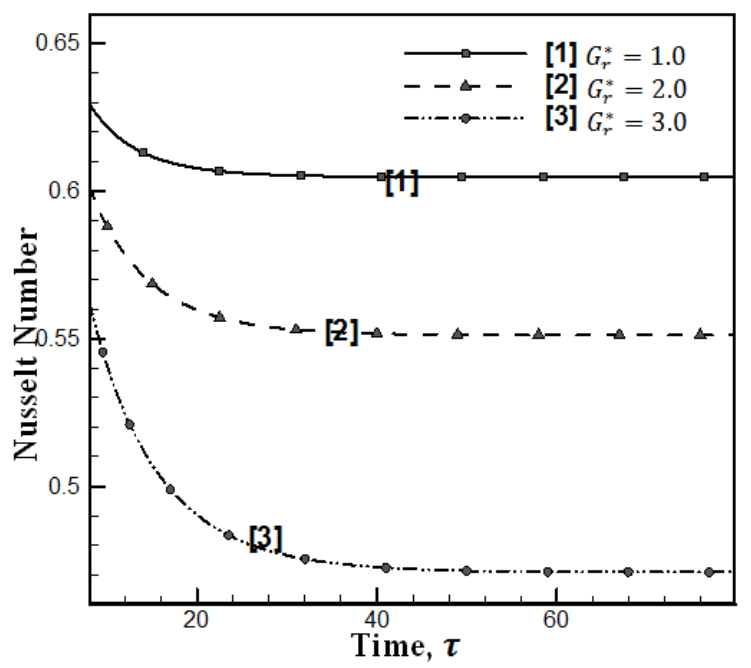

Fig. 27. Nusselt number for different $G^{*}$.

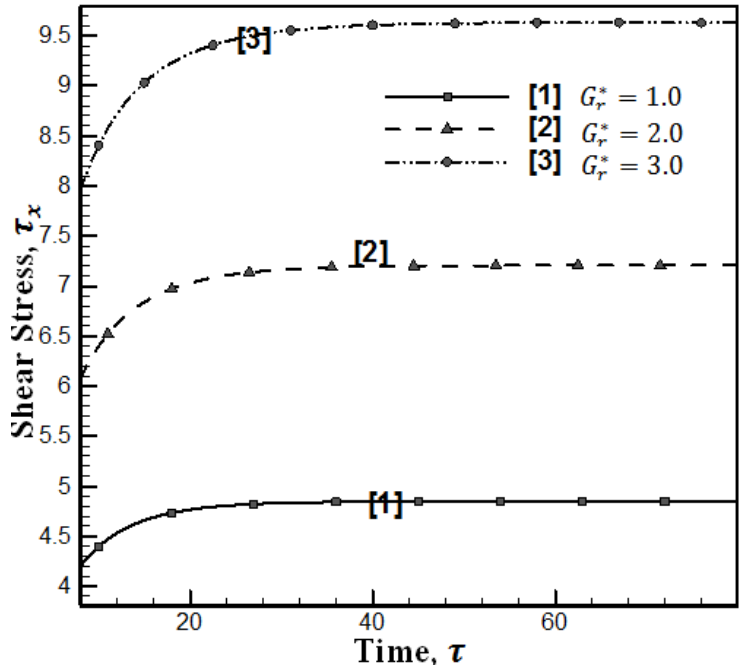

Fig. 24. Primary shear stress for different $G^{*}$.

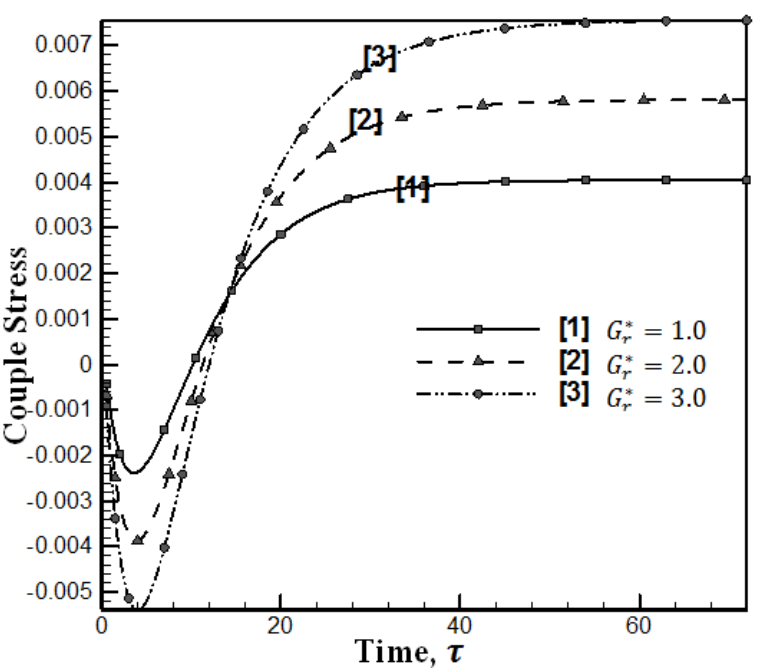

Fig. 26. Couple stress for different $G^{*}$.

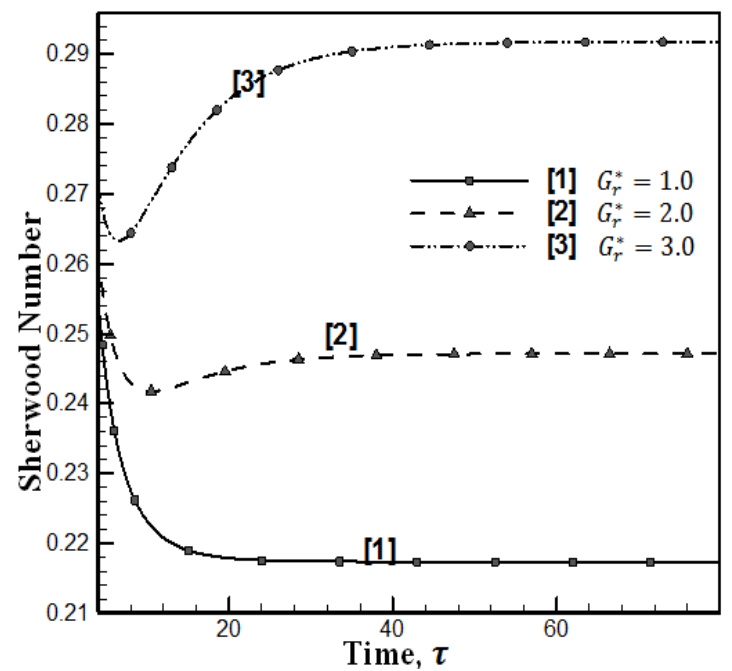

Fig.28. Sherwood number for different $G{ }^{*}$. 


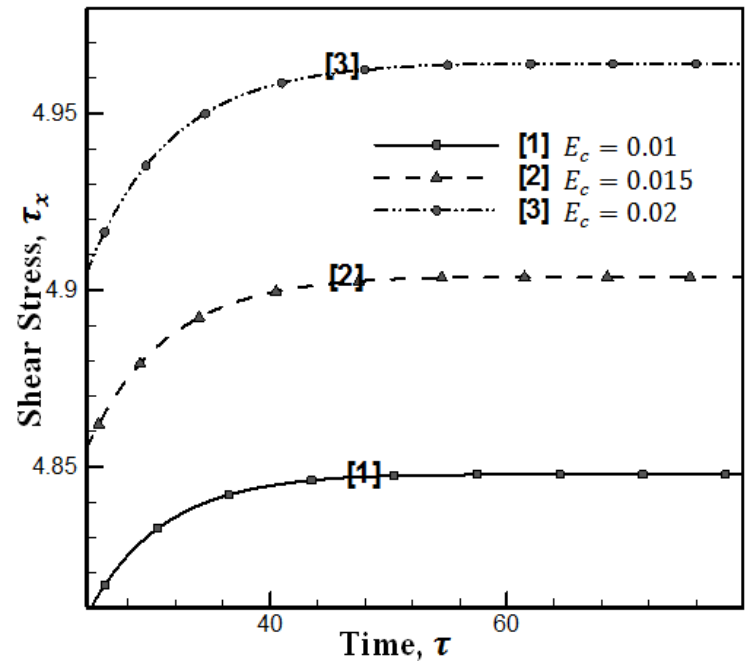

Fig. 29. Primary shear stress with $E_{c}$.

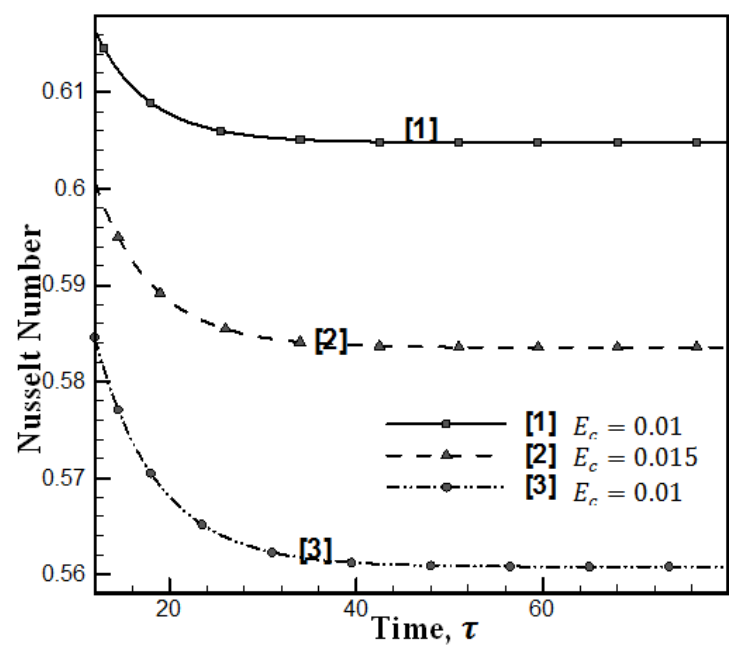

Fig.31. Nusselt number for different $E_{c}$.

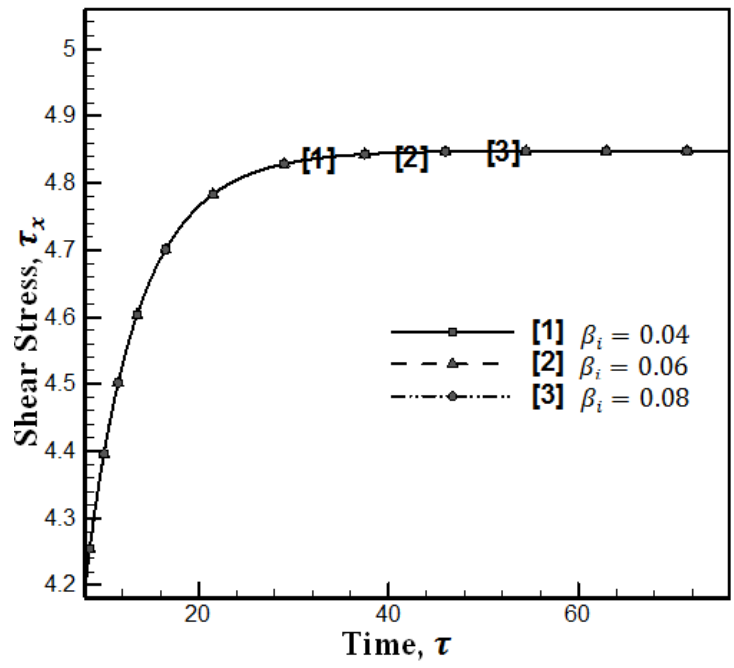

Fig.33. Primary shear stress for ion slip $\left(\beta_{i}\right)$

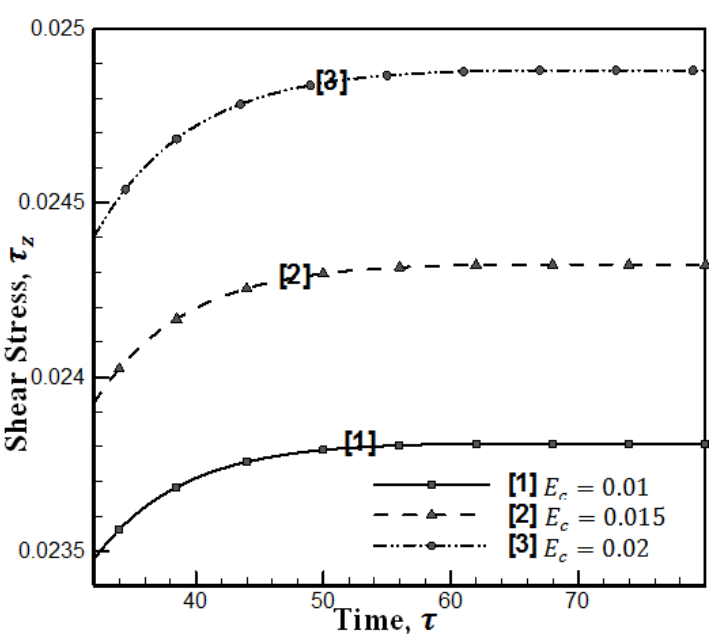

Fig.30. Secondary shear stress with $E_{c}$.

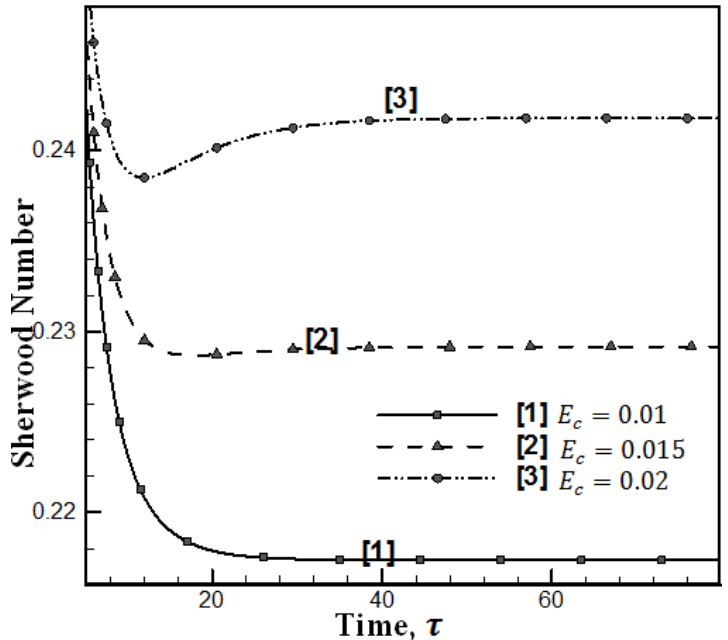

Fig.32. Sherwood number for different $E_{c}$.

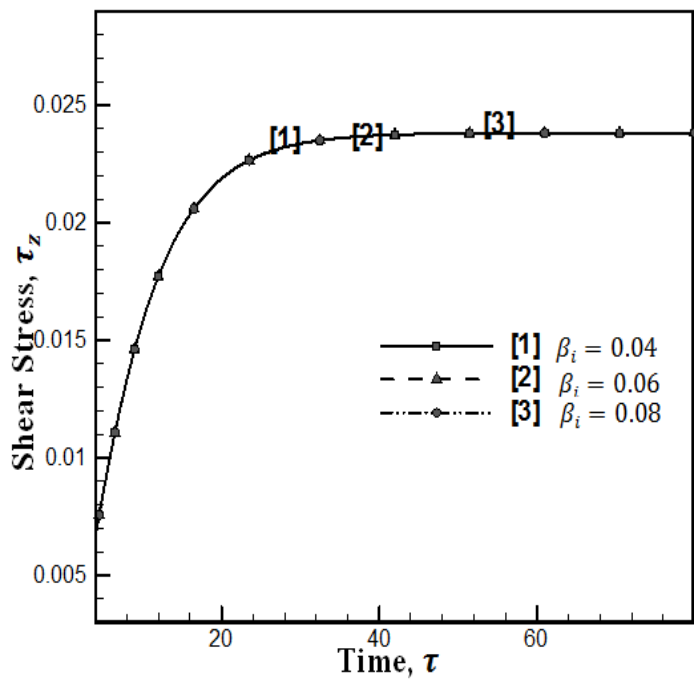

Fig.34. Secondary shear stress for ion slip $\left(\beta_{\mathrm{i}}\right)$ 


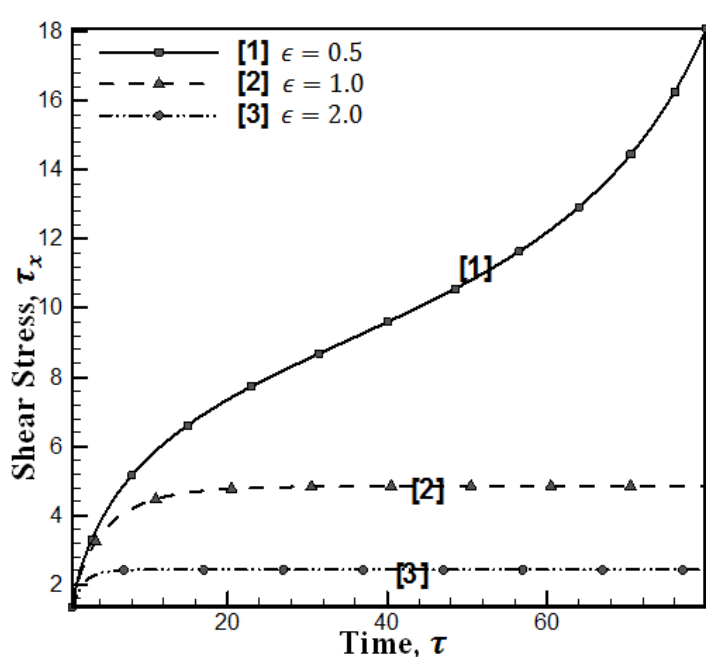

Fig. 35. Primary shear stress with suction $(\varepsilon)$.

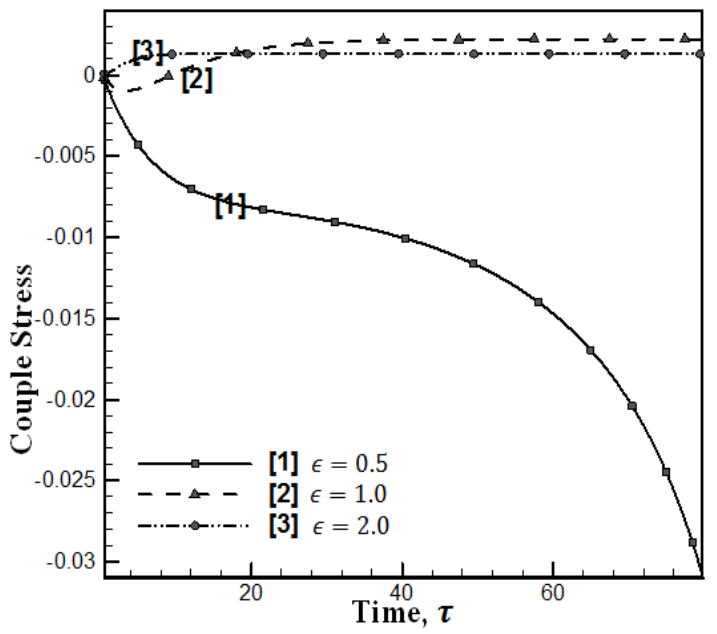

Fig.37. Couple stress with suction $(\varepsilon)$.

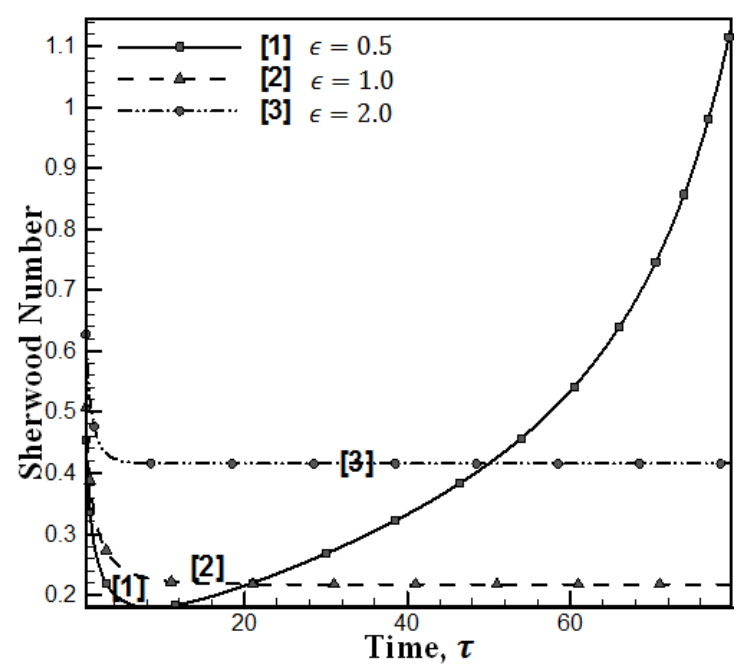

Fig.39. Sherwood number with suction $(\varepsilon)$.

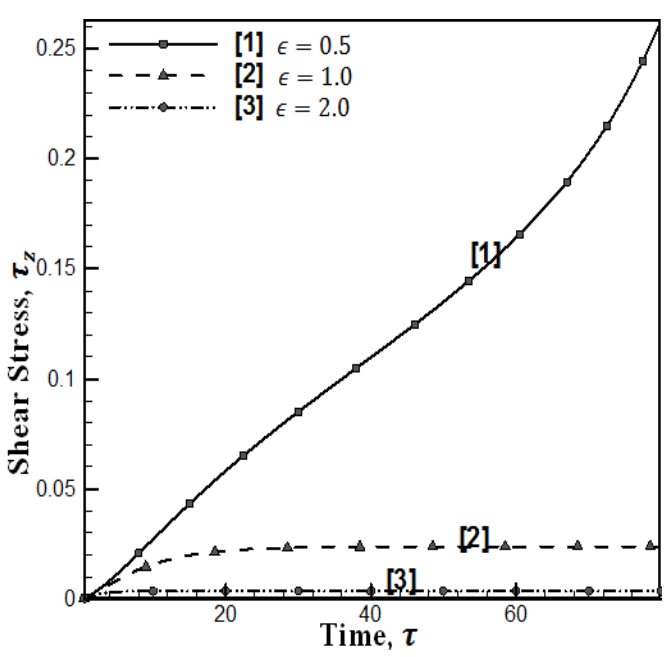

Fig. 36. Secondary shear stress with suction $(\varepsilon)$

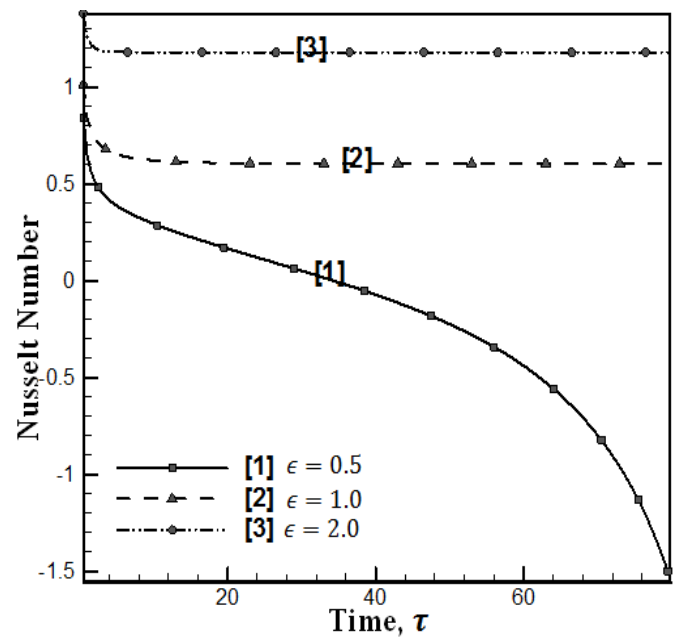

Fig.38. Nusselt number with suction $(\varepsilon)$.

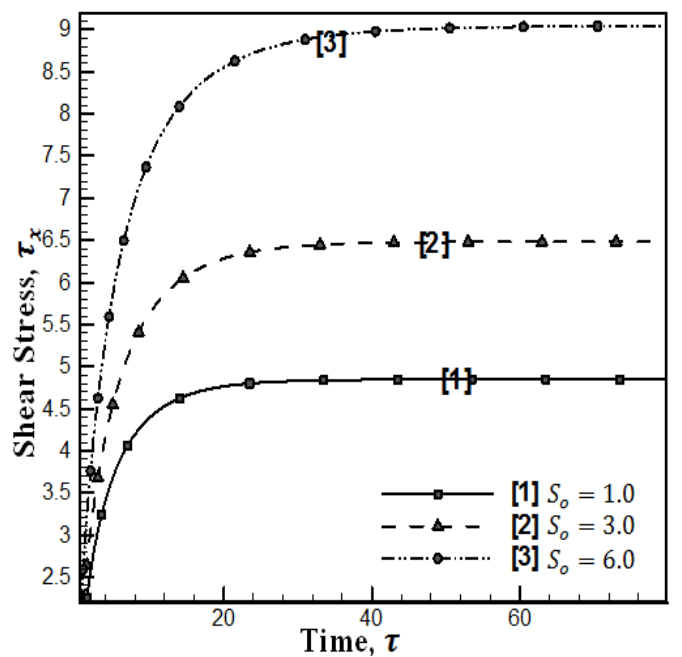

Fig. 40. Primary shear stress with Soret effect $\left(S_{0}\right)$ 


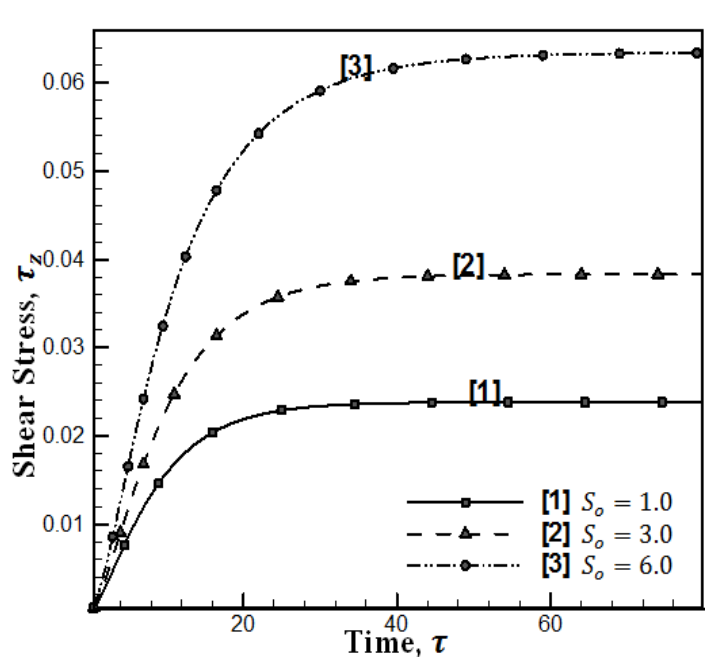

Fig. 41. Primary shear stress with Soret effect

$\left(S_{0}\right)$

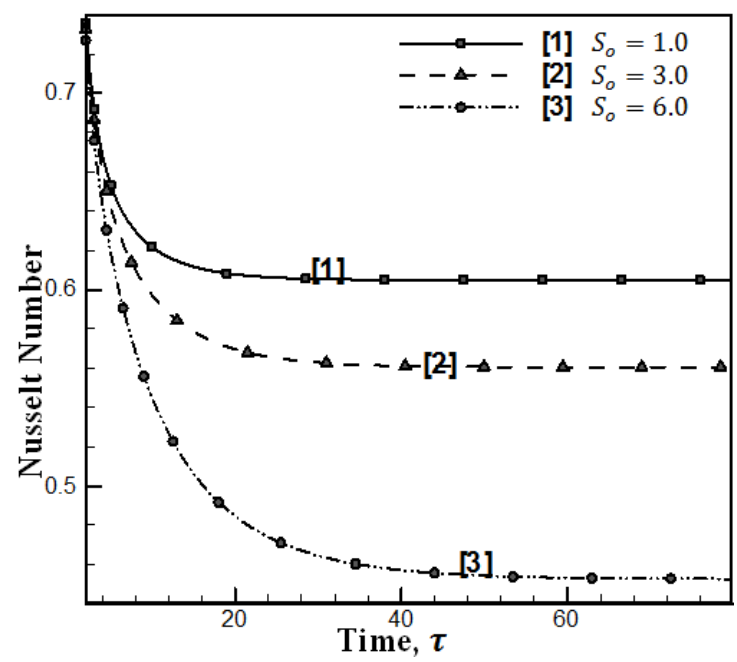

Fig.43. Nusselt number with Soret effect $\left(S_{0}\right)$

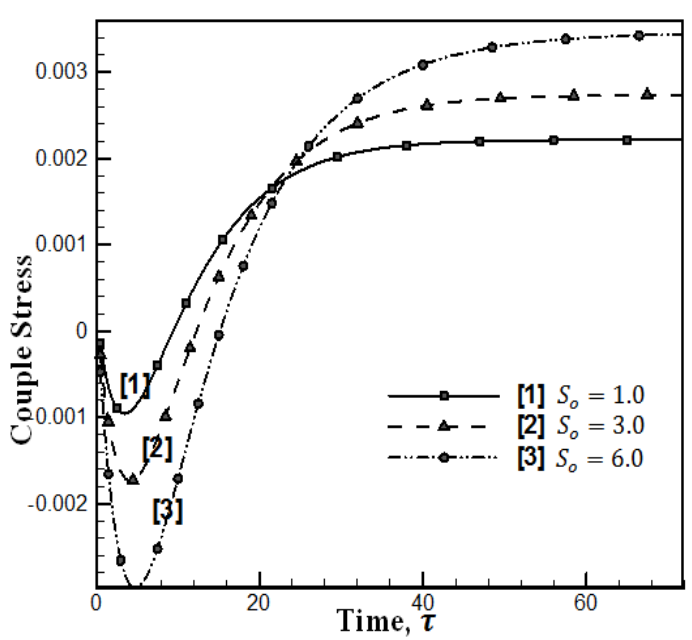

Fig. 42. Couple stress with Soret effect $\left(S_{0}\right)$.

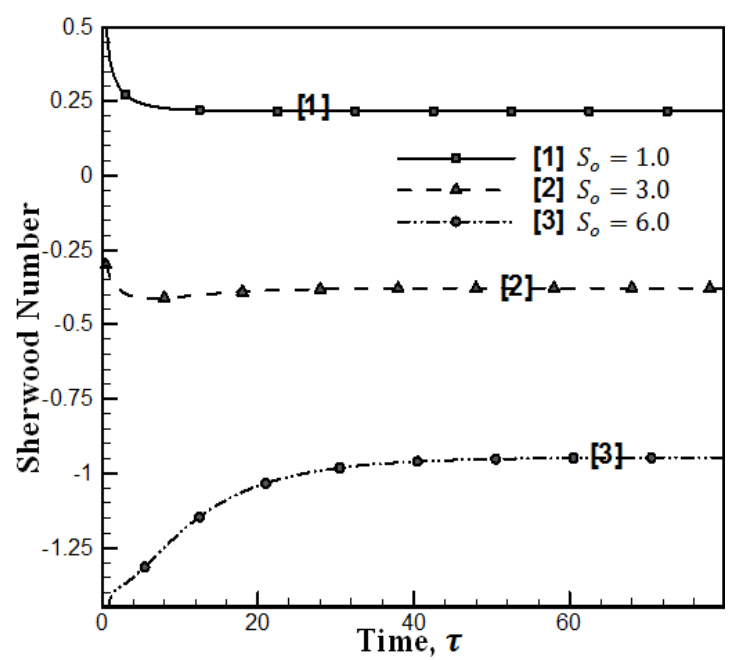

Fig.44. Sherwood number with Soret effect $\left(S_{0}\right)$ 